Aus dem Department für Nutztierwissenschaften

-Abteilung Verfahrenstechnik in der Veredelungswirtschaft-

der Georg-August-Universität Göttingen

Fakultät für Agrarwissenschaften

\title{
Evaluierung verschiedener haltungstechnischer und prozesstechnischer Einflussfaktoren auf das \\ Futteraufnahmeverhalten und die Wachstumsleistung von abgesetzten Ferkeln
}

\author{
Dissertation \\ zur Erlangung des Doktorgrades \\ der Fakultät für Agrarwissenschaften \\ der Georg-August-Universität Göttingen
}

vorgelegt von

Kerstin Reiners

geboren in Delmenhorst

Göttingen, Juli 2009

Die vorliegende Dissertation wurde mit dankenswerter finanzieller Unterstützung der H. Wilhelm Schaumann Stiftung angefertigt 
D 7

Referent:

Prof. Dr. Ir. H. F. A. Van den Weghe

Korreferent:

Prof. Dr. M. Wicke

Tag der mündlichen Prüfung: $\quad$ 16. Juli 2009 


\section{Inhaltsverzeichnis}

Abkürzungsverzeichnis ..............................................................................

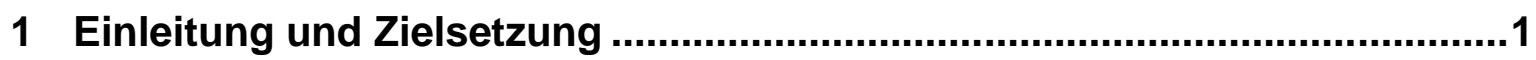

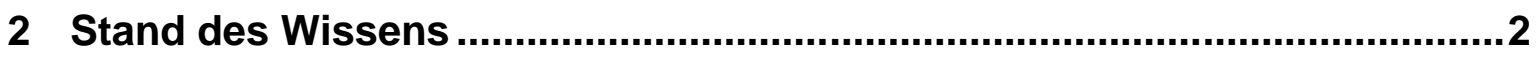

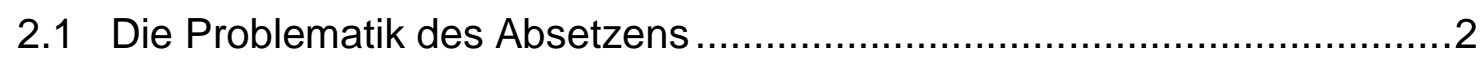

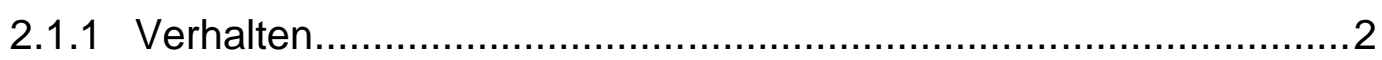

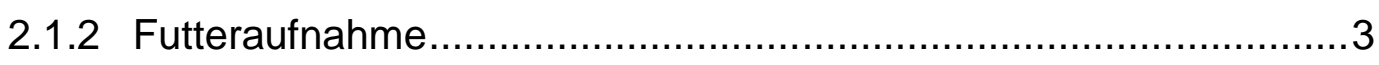

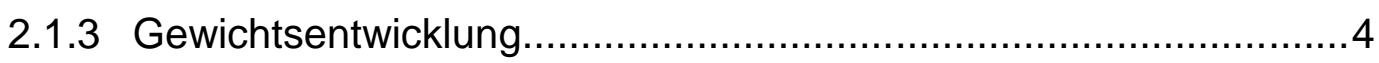

2.2 Einflussfaktoren auf die Futteraufnahme und die Gewichtsentwicklung

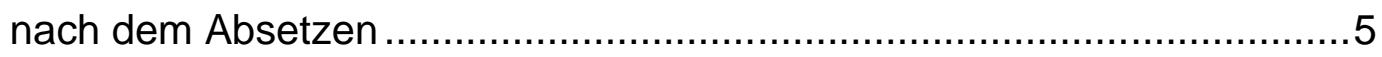

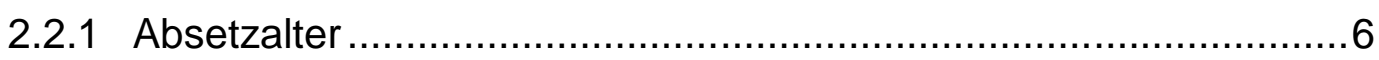

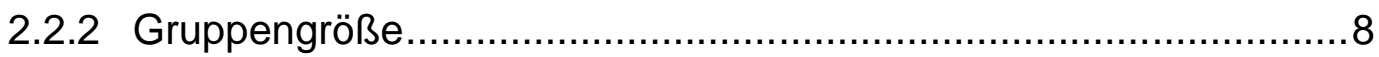

2.2.3 Futterkonsistenz und Futterbereitstellung ..................................

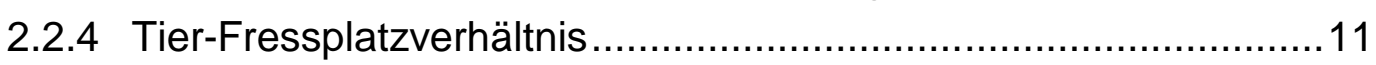

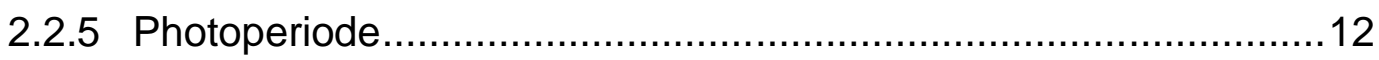

2.2.6 Mischen wurffremder Ferkel...................................................... 14

2.3 Abgeleitete Vorgehensweisen ...................................................... 15

3 Beiträge mit Begutachtung .................................................................. 18

3.1 The effect of heated mash on performance and feeding behavior of newly weaned piglets.

3.2 Application of RFID technology using passive HF transponders for the individual identification of weaned piglets at the feed trough

3.3 Influence of photoperiod on behavior and performance of newly weaned piglets with a focus on feeding behavior, feed consumption and growth

3.4 Socializing piglets before weaning: Effects on behavior of lactating sows, pre- and postweaning behavior, and performance of piglets .........57

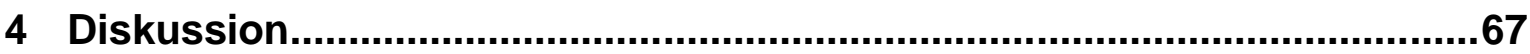

4.1 Evaluierung prozesstechnischer Einflussfaktoren................................67

4.2 Entwicklung einer simultanen Einzeltiererkennung ...............................73

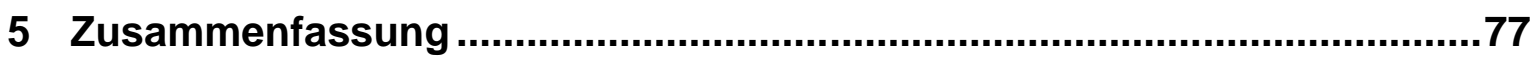

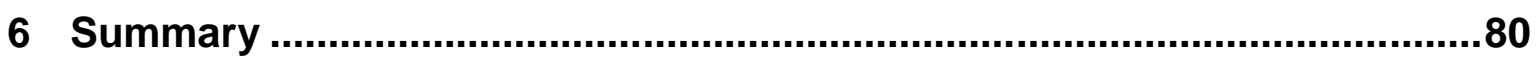

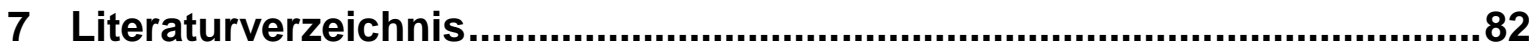

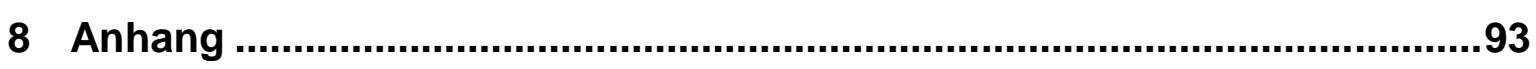

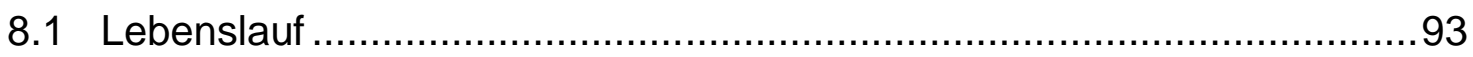

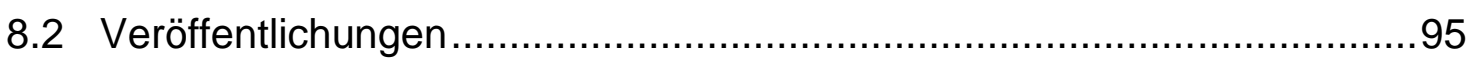

8.2.1 Wissenschaftliche Veröffentlichungen mit Begutachtung..............95

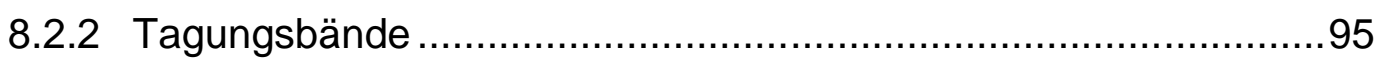

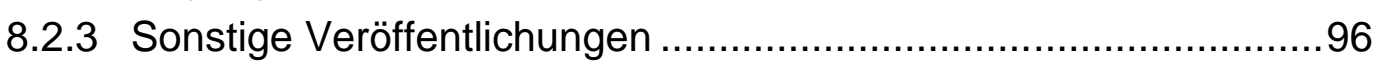




\section{Abkürzungsverzeichnis}

BW

body weight

BWGNP

body weight gain during the nursery period

BWGRP

body weight gain during the rearing period

BWGTP

body weight gain during the total trial period

CFD

calculated farrowing day

CG

control group

CP

crude protein

d

day

DM

dry matter

et al.

und andere / and others

EG

experimental group

EU

Europäische Union

g

Gramm / gram

$\mathrm{G}: F$

gain-to-feed ratio

GLM

general linear model

$\mathrm{h}$

Stunde / hour

HF

Hochfrequenz / high frequency

ID

Identifikation / identification

ISO

Internationale Organisation für Standardisierung /

International Organization for Standardization

$\mathrm{kg}$

Kilogramm / kilogram

$\mathrm{kHz}$

Kilohertz / kilohertz

LF

Niedrigfrequenz / low frequency

LR

longe range

LSM

least square mean

IX

Lux / lux

$\mathrm{m}$

Meter / meter

$\mathrm{m}^{2}$

Quadratmeter / square meter

ME

metebolisierbare Energie / metabolizable energy

$\mathrm{MHz}$

Megahertz / megahertz

Min

Minute / minute

MJ

Megajoule / megajoule 


$\begin{array}{ll}\mathrm{mm} & \text { Millimeter / millimeter } \\ \mathrm{n} & \text { Umfang der Stichprobe / sample size } \\ \mathrm{NS} & \text { nicht signifikant / not significant } \\ \mathrm{P} & \text { Signifikanzniveau / probability } \\ \mathrm{RFID} & \text { Radiofrequenzidentifikation / radio frequency identification } \\ \mathrm{S} & \text { Sekunde / second } \\ \text { SAS } & \text { Statistical Analysis System } \\ \text { SD } & \text { Standardabweichung / standard deviation } \\ \text { SE } & \text { Standardfehler / standard error } \\ \text { SEM } & \text { Standardfehler des Mittelwertes / standard error of the mean } \\ \text { TDMA } & \text { time domain multiple access } \\ \text { V } & \text { Volt / volt } \\ \text { W } & \text { Watt / watt } \\ \text { wk } & \text { week } \\ { }^{\circ} \mathrm{C} & \text { Grad Celsius / degree Celsius } \\ \% & \text { Prozent / percent }\end{array}$




\section{$1 \quad$ Einleitung und Zielsetzung}

Der Grundstein für eine erfolgreiche und damit wirtschaftlich effiziente Schweinemast wird bereits in der Ferkelaufzucht gelegt. Besondere Bedeutung kommt hierbei der Futteraufnahme der Ferkel direkt nach dem Absetzen zu. Durch eine Reihe von Stressoren ist die Futteraufnahme der Tiere nach dem Absetzen reduziert, es kommt zu Wachstumsdepressionen, die weit reichende Folgen für den weiteren Verlauf der Aufzucht, die angeschlossene Mast und die Schlachtleistung der Tiere haben (King und Pluske, 2003; Morméde und Hay, 2003).

In der vorliegenden Arbeit wurden bisher nicht berücksichtigte Einflussfaktoren auf das Futteraufnahmeverhalten und die Wachstumsleistung von abgesetzten Ferkeln analysiert. In vergleichenden Untersuchungen wurde der Einfluss von temperiertem Futterbrei, einer verlängerten Photoperiode in den ersten vier Tagen nach dem Absetzen und einer frühen Sozialisierung der Ferkel bereits vor dem Absetzen evaluiert. Ziel der Untersuchungen war es, durch eine Anpassung verschiedener Faktoren an die natürlichen Verhaltensweisen der Tiere, den Absetzstress zu minimieren und so eine höhere Futteraufnahme und eine daraus resultierende gesteigerte Gewichtsentwicklung der Ferkel nach dem Absetzen zu erreichen.

Im Rahmen der Untersuchungen wurde darüber hinaus mit Hilfe der Radiofrequenzidentifikation (RFID) eine innovative Technik entwickelt und geprüft, mit der es möglich ist, Ferkel simultan individuell am Rundtrog von Futterautomaten zu identifizieren. Diese Technik soll zukünftig aufwändige Videobeobachtungen ablösen, die bisher nötig waren, um den Einfluss verschiedenster Faktoren in Hinblick auf das Tierverhalten und die Tierleistung zu analysieren und miteinander zu vergleichen. Vor dem Hintergrund erhöhter Arbeits- und Verwaltungsanforderungen sowie einer damit einhergehenden steigenden Datenflut wäre es des Weiteren denkbar, das entwickelte System sowohl für die innerbetriebliche Prozesskontrolle als auch für überbetriebliche Kontrollsysteme zu nutzen. RFID-Systeme gelten als Schlüsseltechnologie für das Precision Livestock Farming und werden zukünftig weiter an Bedeutung gewinnen. 


\section{Stand des Wissens}

\subsection{Die Problematik des Absetzens}

Unter natürlichen Bedingungen erfolgt das Absetzen von Ferkeln in einem langsamen Prozess, verbunden mit einer stufenweisen Lockerung der MutterKind-Beziehungen und einer schrittweisen Integration der Ferkel in den Familienverband (Jensen, 1995). Während des Absetzprozesses kommt es zu einer sukzessiven Reduktion der Milchaufnahme, erst nach ca. vier bis fünf Monaten ist die Säugephase vollständig beendet und die Ferkel sind komplett auf die Aufnahme von festem Futter eingestellt (Jensen und Stangel, 1992).

Im Gegensatz zu Wildschweinen und Schweinen, die unter natürlichen Bedingungen gehalten werden, stellt das Absetzen für domestizierte Schweine keinen langsamen Prozess, sondern ein plötzliches Ereignis dar (Brooks und Tsourgiannis, 2003). Mit dem Absetzen ist nicht nur eine abrupte Trennung von der Mutter, sondern auch das Umstallen in eine meist fremde Umgebung, die Konfrontation mit wurffremden Ferkeln, und vor allem eine radikale Futterumstellung von der Sauenmilch zu festem Futter verbunden (Held und Mendl, 2001; Jensen, 2002; King und Pluske, 2003). In diesem Zusammenhang werden hohe Anforderungen an die Anpassungsfähigkeit der Ferkel gestellt. In den meisten Fällen erfolgt die Adaptation an die neue Situation jedoch nicht problemlos, da die Anpassungsfähigkeit der Ferkel überfordert ist. Die Tiere sind einer Reihe von Stressoren ausgesetzt, die weitreichende gesundheitliche, wachstumsverzögernde und damit auch wirtschaftliche Konsequenzen haben können (Morméde und Hay, 2003).

\subsubsection{Verhalten}

Durch das Absetzen kommt es zu einer Reihe von Verhaltensauffälligkeiten. Insbesondere in den ersten Tagen nach dem Absetzen sind die Ferkel sehr unruhig. Es kommt $\mathrm{zu}$ einem erhöhten Auftreten von aggressiven Auseinandersetzungen, da die Tiere in den neu zusammen gesetzten Gruppen gezwungen sind, eine neue Rangordnung $z u$ etablieren (Brooks und Tsourgiannis, 2003; Held und Mendl, 2001; Jensen, 2002; King und Pluske, 2003). Neben dem erhöhten Auftreten von aggressivem Verhalten wird nach dem Absetzen auch ein Anstieg der Vokalisation und Lokomotion der Tiere, 
sowie ein verstärktes gegenseitiges Besaugen am Bauch und an der Flanke beobachtet (Morméde und Hay, 2003; Weary, 2007).

Aus zahlreichen Untersuchungen geht hervor, dass das gegenseitige Besaugen der Tiere als Indikator für ein reduziertes Wohlbefinden herangezogen werden kann. Die Ursache wird darin gesehen, dass die Tiere zum einen versuchen, ihren Hunger zu stillen, zum anderen auch ihren angeborenen Saugdrang zu befriedigen (Fraser und Broom, 2004a; Morméde und Hay, 2003; O'Conell et al., 2004; Tsourgiannis et al., 2004).

Die vermehrte Vokalisation (Grunzen und Quiecken) und Lokomotion (Hochspringen an den Buchtentrennwänden) wird in der Literatur mit Suchlauten, Hilferufen und Hungerlauten in Zusammenhang gebracht und wird daher als ein weiterer Indikator für die enormen Belastungen angesehen, die mit dem Absetzen einhergehen (Held und Mendl, 2001; Hillmann et al., 2001).

\subsubsection{Futteraufnahme}

Eines der Hauptprobleme nach dem Absetzen stellt die reduzierte Futteraufnahme der Ferkel dar. Mit dem Absetzen erfolgt eine abrupte Umstellung von der Sauenmilch auf festes Futter. Die Ferkel müssen die Fähigkeit entwickeln, Futter und Wasser in getrennter Form aufzunehmen und zwischen Hunger und Durst zu unterscheiden (Morméde und Hay, 2003). Teilweise kann es bis zu 50 Stunden dauern, bis $95 \%$ der Ferkel einer Gruppe nach dem Absetzen ihre erste Mahlzeit einnehmen (Bruininx et al., 2001). Aus Untersuchungen von Le Dividich und Séve (2001) geht hervor, dass von Ferkeln am ersten Tag nach dem Absetzen nur noch $25 \%$ der Energie durch festes Futter aufgenommen wird, die vor dem Absetzen durch die Sauenmilch aufgenommen wurde. Im Verlauf der ersten Woche nach dem Absetzen nimmt die Aufnahme von Energie durch festes Futter zwar zu, liegt jedoch noch immer bei nur 60-70 \% dessen, was die Ferkel vor dem Absetzen durch die Sauenmilch aufgenommen haben.

Welche Bedeutung einer frühen und ausreichenden Futteraufnahme von abgesetzten Ferkeln zukommt, beschreiben u. a. Kelly und Coutts (2000), McCracken et al. (1999), Pluske et al. (1996), und Van Beers-Schreurs et al., (1998). Die Autoren sehen in einer verminderten Futteraufnahme der Ferkel unmittelbar nach dem Absetzen den Grund für eine Veränderung der Struktur 
des Dünndarms und die daraus resultierenden, häufig beobachteten Wachstumsdepressionen der Tiere. Die Untersuchungen belegen, dass es durch eine verminderte Futteraufnahme nach dem Absetzen zu einer Athrophie der Darmzotten bei gleichzeitiger Hyperplasie der Krypten kommt. Auf den Darmzotten findet die Absorption der Nährstoffe statt. Bei einer Reduzierung der Zottenoberfläche können folglich nicht mehr ausreichend Nährstoffe in den Organismus gelangen, da sie vermehrt unverdaut in den Dickdarm entlassen werden. Hampson (1986) berichtet, dass die Darmzottenlänge von 21 Tage alten abgesetzten Ferkeln innerhalb der ersten 24 Stunden nach dem Absetzen im Vergleich zur erhobenen Zottenlänge vor dem Absetzen um $75 \%$ reduziert war. Am fünften Tag nach dem Absetzen war die Länge der Darmzotten wieder auf $50 \%$ der Zottenlänge vor dem Absetzen angewachsen. Nicht abgesetzte Tiere zeigten im gleichen Zeitraum keine Athrophie der Darmzotten. Hedemann et al. (2003) bestätigen die Ergebnisse von Hampson (1986). Die Autoren untersuchten den Einfluss des Absetzens auf die Morphologie des Dünndarms im Zeitraum von drei Tagen vor dem Absetzen bis neun Tagen nach dem Absetzen. Die Darmzottenhöhe und die Kryptentiefe wurden an drei Stellen entlang des Dünndarms untersucht. Nach dem Absetzen verkürzten sich die Darmzotten an den rumpfwärts gelegenen Abschnitten des Dünndarms, die minimale Länge wurde am dritten Tag nach dem Absetzen beobachtet. Im hinteren Abschnitt des Dünndarms nahm die Zottenhöhe nicht ab. Die Kryptentiefe wiederum war am dritten bis siebten Tag nach dem Absetzen an allen untersuchten Stellen des Dünndarms erhöht. Die größten Veränderungen konnten am dritten Tag nach dem Absetzen beobachtet werden. In den folgenden Tagen wurde eine graduelle Rekonstruierung des Dünndarms festgestellt. Auch Pluske et al. (1997) konnten in ihren Untersuchungen belegen, dass die Länge der Darmzotten von der Höhe der Futteraufnahme nach dem Absetzen beeinflusst wird. Die Autoren halten eine kontinuierliche Versorgung der Ferkel mit Nährstoffen nach dem Absetzen für unerlässlich, um die Zottenstruktur des Darmes auch nach dem Absetzen zu erhalten.

\subsubsection{Gewichtsentwicklung}

Das Wachstumspotential von abgesetzten Ferkeln ist grundsätzlich sehr hoch. Im Abschnitt zwischen 10 und $25 \mathrm{~kg}$ Lebendgewicht können bis zu $450 \mathrm{~g}$ 
Tageszunahmen erreicht werden (Jeroch et al., 1999). Whittemore (1998) konnte in Untersuchungen feststellen, dass gesunde Ferkel mit einem Absetzgewicht von etwa $5 \mathrm{~kg}$ bei einer nicht restriktiven Fütterung sogar ein potentielles Wachstumsvermögen von bis zu $500 \mathrm{~g}$ pro Tag haben. Bedingt durch die reduzierte Futteraufnahme der Ferkel nach dem Absetzen und die daraus resultierende Rückbildung der Darmzotten kommt es in den ersten Wochen nach dem Absetzen jedoch häufig zu Wachstumsdepressionen, die weit reichende Folgen für den weiteren Verlauf der Aufzucht und die nachfolgende Mast der Tiere haben (King und Pluske, 2003). Von welcher Bedeutung die Zunahmen in den ersten Wochen nach dem Absetzen sind und wie diese das Wachstum der Tiere bis zur Schlachtung beeinflussen, machen Pluske et al. (1995) deutlich. In ihren Untersuchungen konnten sie feststellen, dass Ferkel, die in der ersten Aufzuchtswoche Tageszunahmen von über $115 \mathrm{~g}$ hatten, das Schlachtgewicht etwa 14 Tage früher erreichten als Ferkel, deren tägliche Zunahmen in der ersten Aufzuchtswoche bei unter $100 \mathrm{~g}$ lagen. Auch Williams (2003) untersuchte den Einfluss der Gewichtsentwicklung der Ferkel in der ersten Woche nach dem Absetzen auf die Schlachtreife. Die Untersuchungen ergaben, dass Ferkel, die in der ersten Aufzuchtswoche eine tägliche Zunahme von $225 \mathrm{~g}$ hatten, bei der Schlachtung am 156. Tag $8 \mathrm{~kg}$ schwerer waren als die Ferkel, die inr Absetzgewicht in der ersten Woche der Aufzucht beibehielten.

\subsection{Einflussfaktoren auf die Futteraufnahme und die Gewichtsentwick- lung nach dem Absetzen}

Der Einfluss verschiedenster Einflussfaktoren auf die Futteraufnahme und die Wachstumsleistung von Ferkeln wurde in zahlreichen Untersuchungen analysiert. So sehen beispielsweise Wellock et al. (2003) die Gruppengröße, das Platzangebot, das Tier-Fressplatzverhältnis und das Mischen wurffremder Ferkel nach dem Absetzen als Hauptursachen für die verminderte Futteraufnahme der Ferkel nach dem Absetzen. Zijlstra und Scott (2000) hingegen unterteilen die Stressoren, die die Futteraufnahme und damit die Wachstumsleistung von Ferkeln beeinflussen können in Umwelteinflüsse, soziale Einflüsse (Gruppengröße, Gruppenzusammenstellung) und immunologische Einflüsse. 
Untersuchungen an Hausschweinen in natürlicher Umgebung haben ergeben, dass die meisten Verhaltensweisen domestizierter Hausschweine denen des europäischen Wildschweines entsprechen (Jensen, 2002; Stolba und WoodGush, 1989). Die durch die Domestikation und die Züchtung erfolgte Veränderung des genetischen Materials der Hausschweine führte demnach nicht zu einer Veränderung der Verhaltensweisen (Stolba und Wood-Gush, 1989).

Um eine Futteraufnahme der Ferkel unmittelbar nach dem Absetzen und damit hohe Gewichtszunahmen in den ersten Aufzuchttagen erreichen zu können, sollten die Haltungs- und Fütterungssysteme der Tiere so weit wie möglich an die Ansprüche der Tiere angepasst sein (Brooks und Tsourgiannis, 2003; Madec et al., 2003).

Auch Weary et al. (2007) sehen in der Optimierung der Haltungs- und Fütterungssysteme in Bezug auf eine Anpassung an die natürlichen Verhaltensweisen eine Möglichkeit, die Tiergerechtheit und damit die Effizienz der Ferkelaufzucht zu verbessern. Die Autoren weisen darauf hin, dass eine Reduktion der Stressoren nach dem Absetzen eine deutliche Verringerung der Stressantwort der Tiere nach dem Absetzen bewirken kann.

Im Folgenden werden einige ausgewählte Faktoren behandelt, die den Prozess des Absetzens und damit die Wirtschaftlichkeit der Schweineproduktion beeinflussen.

\subsubsection{Absetzalter}

Unter natürlichen Bedingungen erfolgen der Abnabelungsprozess von der Sau und damit die Umstellung von der Sauenmilch auf festes Futter in einem stufenweisen Prozess. Je älter die Ferkel werden, desto unabhängiger werden sie von der Sau und desto mehr trockenes Futter wird von den Tieren konsumiert (Jensen und Recén, 1989).

Vor dem Hintergrund dieses natürlichen Absetzprozesses sehen Miller et al. (2007) und Weary et al. (2007) im Absetzalter der Ferkel einen der Haupteinflussfaktoren auf die Futteraufnahme und damit das Wachstum der Tiere nach dem Absetzen. In zahlreichen Untersuchungen konnte nachgewiesen werden, dass die Futteraufnahme der Ferkel nach dem Absetzen positiv mit dem Absetzalter korreliert. 
So konnten Dritz et al. (1996) in Untersuchungen zeigen, dass die Futteraufnahme nach dem Absetzen bei Ferkeln mit einem Absetzalter von 19 Tagen höher war als bei Ferkeln, die bereits nach neun Tagen von der Mutter getrennt wurden. Diese Ergebnisse werden von Gonyou et al. (1998) bestätigt, die nachweisen konnten, dass Ferkel mit einem Absetzalter von zwölf Tagen in den ersten 48 Stunden nach dem Absetzen nur halb so viel Zeit mit der Futteraufnahme verbrachten, wie Ferkel, die mit 21 Tagen abgesetzt wurden. Colson et al. (2006) konnten feststellen, dass die Wachstumsdepressionen bei Ferkeln, die mit 28 Tagen abgesetzt wurden im Vergleich zu Ferkeln, die bereits mit 21 Tagen abgesetzt wurden, halbiert waren.

Miller et al. (2007) begründen den positiven Einfluss des höheren Absetzalters auf das Wachstum der Tiere mit der Entwicklung der Darmzotten. Ferkel mit einem Absetzalter von sechs Wochen zeigten längere Darmzotten und eine höhere Kryptentiefe als Ferkeln, die bereits mit vier Wochen abgesetzt wurden.

Der Einfluss des Absetzalters und damit des Absetzgewichts spiegelt sich neben der Aufzuchtsleistung auch in der Mast- und Schlachtleistung der Tiere wider. Mahan und Lepine (1991) konnten in ihren Untersuchungen zeigen, dass Tiere mit einem erhöhten Absetzgewicht diesen Vorteil bis zum Zeitpunkt der Schlachtung beibehielten. Diese Ergebnisse bestätigen Untersuchungen von Lawlor et al. (2002), die eine Korrelation zwischen dem Absetzgewicht und der Streuung der Gewichtszunahmen nach dem Absetzen nachweisen konnten. Je höher das Absetzgewicht war, desto geringer waren die Streuungen der Gewichtszunahmen innerhalb der Gruppen.

Bei der Bestimmung des optimalen Absetzalters muss jedoch neben der Futteraufnahme und der Wachstumsleistung auch der Einfluss des Absetzalters auf das Aggressionsverhalten der Ferkel berücksichtigt werden. Es gibt Annahmen darüber, dass das Aggressionsverhalten der Ferkel sich mit zunehmendem Alter ändert (Held und Mendl, 2001). So konnte beispielsweise Jensen (1994) in seinen Untersuchungen beobachten, dass die Kämpfe zur Etablierung einer Rangordnung zwischen Ferkeln im Alter von einer Woche kürzer waren als zwischen Ferkeln, die in einem Alter von fünf oder neun Wochen gemischt wurden. Jensen (1994) vermutete, dass jüngere Ferkel weniger Motivation hatten zu kämpfen, dass ihnen weniger Energie für das Austragen von langen Kämpfen zur Verfügung stand, oder dass sie in der Lage 
waren, schneller eine soziale Hierarchie auszubilden als ältere Ferkel. Zu vergleichbaren Ergebnissen kamen auch Pitts et al. (2000), die in ihren Untersuchungen beobachteten, dass Ferkel, die im Alter von 26 Tagen mit fremden gemischt wurden, insgesamt länger kämpften und damit auch mehr Verletzungen zeigten als Ferkel, die im Alter von fünf Tagen gemischt wurden. Die Autoren dieser Untersuchung nahmen an, dass die Toleranzgrenze jüngerer Ferkel gegenüber wurffremden Ferkeln höher ist als die von Ferkeln höheren Alters.

\subsubsection{Gruppengröße}

Die Haltung von abgesetzten Ferkeln in Großgruppen (> 100 Tiere) gewinnt in der konventionellen Schweinehaltung zunehmend an Bedeutung. Speziell in den USA und in Australien wird bereits seit einigen Jahren mit sogenannten Megagruppen gearbeitet (Madec et al., 2003). Aufgrund der Forderung nach immer größeren, einheitlichen Verkaufsgruppen wird die Großgruppenhaltung von abgesetzten Ferkeln auch in Deutschland seit einigen Jahren diskutiert (Weber, 2004).

Bei der Haltung von Ferkeln in Großgruppen kann das individuelle Platzangebot pro Ferkel effektiver genutzt werden und die Trennung der Bucht in verschiedene Funktionsbereiche (Aktivitätsbereich, Ruhebereich und Kotbereich) wird möglich (von Borell et al., 1997). Vor dem Aspekt der Sauberkeit und damit der Tierhygiene sind Großgruppen vorteilhaft, da weniger Buchtenabtrennungen nötig sind als bei kleineren Gruppen. Somit ist der Anteil der Kotfläche bei größeren Gruppen geringer. Neben dem ökonomischen Vorteil von Großgruppen durch verringerte Investitionskosten, liegt der Nachteil von Großgruppen in einer erschwerten Tierbeobachtung (Spoolder et al., 1999). Vor dem Hintergrund der Tiergerechtheit, bringen Großgruppen in der Ferkelaufzucht Vorteile mit sich. Aufgrund des effektiveren Platzangebotes werden den Tieren Ausweichmöglichkeiten geschaffen, die in einem im Vergleich zur Kleingruppe reduziertem Aggressionsverhalten der Tiere, v. a. nach dem Absetzen, resultieren (Gonyou, 2001). Kircher (2001) verglich das Aggressionsverhalten von Ferkeln, die in einer 40er Gruppe gehalten wurden mit dem Aggressionsverhalten von Ferkeln, die in einer 60er Gruppe gehalten wurden. In den 40er Gruppen traten im Vergleich zu den Gruppen, in denen 60 
Ferkel gehalten wurden, vermehrt Aggressionen auf. Die Autorin nimmt an, dass sich die Ferkel in der 40er Gruppe gegenseitig nicht mehr kennen. In der 60er Gruppe hingegen bildeten sich Untergruppen, die getrennt den Trog aufsuchten und sich deshalb nicht behinderten. Auch Gonyou (2001) vermutet, dass sich in großen Gruppen sogenannte Untergruppen bilden. Des Weiteren nimmt er an, dass die Tiere größerer Gruppen eine Toleranz gegenüber anderen Tieren ausbilden.

Im Gegensatz zu den Untersuchungen von Kircher et al. (2001) und Gonyou (2001) konnten Weber et al. (2002) in vergleichenden Untersuchungen mit Gruppengrößen von zehn, 20 oder 40 Ferkeln pro Bucht keinen Einfluss der Gruppengröße auf die Aggressionen der Tiere nach dem Absetzen feststellen. Bezüglich des Einflusses der Gruppengröße auf das Futteraufnahmeverhalten und die Wachstumsleistung der Ferkel liegen unterschiedliche Erkenntnisse vor. So konnten bspw. McConell (1987) bereits in frühen Untersuchungen über die gesamte Aufzuchtperiode hinweg bei gleichbleibendem Platzangebot pro Tier bei steigender Gruppengröße eine Reduktion der Futteraufnahme und der täglichen Zunahmen beobachten. Diese Annahmen konnten in späteren Untersuchungen von Wolter und Ellis (2002) bestätigt werden. Bei einer Steigerung der Gruppengröße von 20 auf 100 Tiere konnte hier bei der Futteraufnahme ein Rückgang zwischen $4,3 \%$ und 6,6 \% beobachtet werden. Bei den täglichen Zunahmen variierte der Rückgang zwischen 5,1 \%und 6,6 \%. Diese Ergebnisse werden von Wolter et al. (2000) bestätigt.

Im Gegensatz zu McConell (1987) und Wolter und Ellis (2002) konnten O'Connell et al. (2001) in einer Untersuchung, in der insgesamt 1280 Ferkel in Gruppen von 10, 20, 30, 40 und 60 Tieren nach dem Absetzen für 28 Tage beobachtet wurden, keinen Einfluss der Gruppengröße auf das Futteraufnahmeverhalten und das Wachstum der Tiere feststellen. Auch Gonyou (1999) konnte in Gruppen von 100 Ferkeln die gleiche Produktivität wie bei Gruppengrößen von 20 bis 40 Ferkeln nachweisen.

\subsubsection{Futterkonsistenz und Futterbereitstellung}

Die Futteraufnahme von Ferkeln nach dem Absetzen wird in hohem Maße von der Schmackhaftigkeit und der Konsistenz des Futters bestimmt (Brooks und Tsourgiannis, 2003). Während in der Ferkelaufzucht bis vor wenigen Jahren 
noch der Trockenfutterautomat dominierte, gewinnen Breifutterautomaten und Flüssigfütterungssysteme für die Ferkelaufzucht immer mehr an Bedeutung (Weber, 2004). Flüssiges, feucht-krümeliges bis breiiges Futter wird im Vergleich zu mehlförmigem oder pelletiertem Trockenfutter von den Ferkeln bevorzugt. In Untersuchungen von Kim et al. (2001) konnte nachgewiesen werden, dass Ferkel, die nach dem Absetzen flüssiges oder breiförmiges Futter erhielten, eine höhere Futteraufnahme zeigten als Ferkel, die trockenes Futter erhielten. Auch Partridge et al. (1992) konnten in früheren Untersuchungen zeigen, dass Ferkel, die breiförmiges Futter erhielten, $13 \%$ mehr Futter aufnahmen und $11 \%$ schneller wuchsen als Ferkel, die mit pelletiertem trockenen Futter gefüttert wurden. Als Grund wurde hier angenommen, dass das breiförmige Futter der Sauenmilch ähnlicher und so für die Ferkel attraktiver ist. Diese Ergebnisse konnten von Hessel und Van den Weghe (2005) bestätigt werden. Hier zeigten Ferkel, die mit breiförmigem Futter gefüttert wurden, über die gesamte Aufzuchtperiode hinweg Mehrzunahmen von durchschnittlich $840 \mathrm{~g}$. Die Futteraufnahme war bei den Ferkeln, die breiförmiges Futter erhielten, ebenfalls erhöht.

Im Gegensatz zu Kim et al. (2001) und Hessel und Van den Weghe (2005) konnten Kornegay und Thomas (1981) in früheren Untersuchungen keinen Einfluss der Futterkonsistenz auf die tägliche Futteraufnahme und die Gewichtsentwicklung von abgesetzten Ferkeln feststellen. Auch Lawlor et al. (2002) konnten in den ersten 28 Tagen nach dem Absetzen keinen positiven Einfluss von flüssigem Futter auf die Futteraufnahme und die Wachstumsleistung der Ferkel nachweisen.

In zahlreichen Untersuchungen konnte gezeigt werden, dass die Futteraufnahme der Ferkel nach dem Absetzen neben der Futterkonsistenz auch von der Bereitstellung des Futters bestimmt wird. Zum Teil scheitert die Futteraufnahme daran, dass die Ferkel keinen ausreichenden Zugang zum Futter haben oder dass das Erkundungs- und Futteraufnahmeverhalten noch nicht weit genug fortgeschritten ist, um die Fütterungssysteme entsprechend bedienen zu können (bspw. Hebelbewegungen an Futterautomaten, um Futter mit Wasser zu Brei zu vermengen). Um eine optimale Futterversorgung gewährleisten zu können, kommt der Gestaltung der Fütterungssysteme daher eine entscheidende Bedeutung zu (Brooks und Tsourgiannis, 2003; Fraser und 
Broom, 2004b). So hat es sich beispielsweise bewährt, das Futter stets frisch zu füttern. Hohe Fütterungsfrequenzen und kleine Portionen mit flüssiger oder breiiger Konsistenz erhöhen die Futteraufnahme und können die Neugierde der Ferkel wecken (Pajor et al., 1991). Eine gute Vorbereitung auf die Fütterung lässt sich weiterhin auch durch Signale erreichen, die bereits kurz vor der Fütterung einsetzen. Derartige Futtersignale stimulieren die Aktivität der Verdauungssäfte und lösen eine erhöhte Futterappetenz aus (Grauvogel, 1970; von Zerboni und Grauvogel, 1984).

Kojima et al. (2007) sehen die Ursache einer verminderten Futteraufnahme nach dem Absetzen in der Expression von appetit-zügelnden Genen. Nach Meinung der Autoren ist die Expression dieser Gene jedoch weniger von der Darreichungsform des Futters nach dem Absetzen, sondern eher von den Bedingungen, denen die Ferkel vor dem Absetzen ausgesetzt waren, abhängig. Diese Annahme wird von Weary et al. (2007) unterstützt, die in einer zu geringen Aufnahme von festem Futter vor dem Absetzen die Ursache für die reduzierte Futteraufnahme nach dem Absetzen sehen. Ferkel, die vor dem Absetzen neben der Sauenmilch kein festes Futter konsumierten, zeigten nach dem Absetzen im Vergleich zu Ferkeln des gleichen Wurfes, die bereits vor dem Absetzen festes Futter konsumierten, ein erhöhtes abnormales Verhalten in Form von gegenseitigem Besaugen und einer erhöhten Vokalisation. Darüber hinaus konnte ein erhöhter Cortisolspiegel nachgewiesen werden (Mason et al., 2003). Auch Bruininx et al. (2002a) konnten in ihren Untersuchungen nachweisen, dass eine erhöhte Aufnahme von festem Futter vor dem Absetzen die Futteraufnahme nach dem Absetzen positiv beeinflusst.

\subsubsection{Tier-Fressplatzverhältnis}

Die Sicherstellung einer ausreichenden Anzahl an Fressplätzen stellt eine der Grundvoraussetzungen für eine hohe Futteraufnahme der Ferkel nach dem Absetzen dar. In Abhängigkeit vom Fütterungssystem steht das TierFressplatzverhältnis in engem Zusammenhang mit dem Tierverhalten und der Tierleistung (Brooks und Tsourgiannis, 2003). Je mehr Ferkel an einem Futterautomaten fressen, desto geringer wird die Anzahl der Mahlzeiten pro Tier. Bei früheren Futterautomaten mit einem Tier:Fressplatzverhältnis von 1:1 war es nicht unüblich, dass Ferkel bis zu 120 Minuten pro Tag mit dem Fressen 
verbrachten. Bei heutigen Systemen, bei denen sich bis zu 20 Ferkel einen Fressplatz teilen, verbringen die Tiere ca. $40 \%$ weniger Zeit mit der Futteraufnahme (Gonyou, 1999).

Lexer et al. (2001) stellten in ihren Untersuchungen fest, dass die Etablierung des arttypischen biphasischen Aktivitätsrhythmus bei einer zu geringen Anzahl von Fressplätzen beeinträchtigt und die nächtliche Ruhephase dadurch erheblich verkürzt wird. Bei einer zu hohen Belegung der Futterautomaten besteht daher die Gefahr, dass die Fresszeiten der Tiere sich vermehrt in die Nachtstunden verschieben und die Verdrängungen vom Trog zunehmen (Turnert et al. 2002). Auch Georgsson und Svendsen (2002) konnten in ihren Untersuchungen zeigen, dass ein engeres Tier-Fressplatzverhältnis vor dem Hintergrund der Tiergerechtheit sinnvoller ist.

Schlichte (1999) konnte einen Einfluss des Tier-Fressplatzverhältnisses auf die Tageszunahmen feststellen. Bei einem Vergleich von TierFressplatzverhältnissen von 5:1 mit Tier-Fressplatzverhältnissen von 6:1 führte das engere Tier-Fressplatzverhältnis zu höheren Tageszunahmen. Auch in Untersuchungen von Schäfer (1999), in denen ein Tier-Fressplatzverhältnis von 4:1, 5:1 und 7:1 bei unterschiedlicher Gruppengröße untersucht wurde, wurden bei einem weiteren Tier-Fressplatzverhältnis geringere Zunahmen beobachtet. Kirchner (2001) setzte in ihren Untersuchungen einen Rohrbreiautomaten mit sechs Fressplätzen sowohl bei einer Gruppe mit 40 Tieren also auch bei einer Gruppe mit 60 Tieren ein. Hieraus ergab sich ein Tier-Fressplatzverhältnis von $6,7: 1$ und 10:1. Auch in dieser Untersuchung waren die täglichen Zunahmen bei einem Tier-Fressplatzverhältnis von 10:1 geringer als bei einem TierFressplatzverhältnis von 6,7:1. Sowohl Kircher (2001) als auch Schäfer (1999) weisen jedoch darauf hin, dass bei einer Beurteilung von TierFressplatzverhältnissen sowohl die Gruppengrößen als auch die angebotenen Fütterungssysteme berücksichtigt werden müssen (s. Kapitel 2.2.2 und 2.2.3).

\subsubsection{Photoperiode}

In der Literatur wird hinsichtlich der Aktivität und damit auch des Futteraufnahmeverhaltens von Hausschweinen von einem endogen angelegten biphasischen Aktivitätsrhythmus vom Alternanstyp berichtet, der in einem Zeitraum von acht bis zehn Stunden abläuft. Hauptzeitgeber ist hierbei der Hell- 
Dunkelwechsel (Achebe, 1975; Braun und Marx, 1994; Buchholz, 1990, Duven, 1991; Marx et al. 1988; Schrenk und Marx, 1982). Unter natürlichen Lichtverhältnissen zeichnen sich zwei Hauptaktivitätsphasen, die in der Morgenund in der Abenddämmerung liegen und von einer mittäglichen Ruhepause unterbrochen sind, ab. Dem abendlichen Aktivitätsmaximum schließt sich etwa zwei Stunden nach Sonnenuntergang eine nächtliche Ruhepause an. Bei abnehmender Lichtzeit werden die Ruhephasen in der Tagesmitte reduziert, bis im Winter die morgendliche und abendliche Aktivitätsphase miteinander verschmelzen (Duven, 1991; Van Putten, 1978). Versuche von Lexer et al. (2001) sowie Lehmann und Weber (2005) und Schrenk (1981) bestätigen auch bei Ferkeln einen biphasischen Tagesrhythmus.

Der Einfluss des Lichts auf den biphasischen Aktivitätsrhythmus von Schweinen konnte nicht nur unter natürlichen Bedingungen, sondern auch in fensterlosen Ställen unter Einsatz eines Beleuchtungsprogrammes nachgewiesen werden (Bigelow und Houpt, 1988; Bruininx et al., 2002b; Marx et al., 1988; Niekamp et al., 2007). Der Grund für den Einfluss des Lichts auf den Aktivitätsrhythmus der Tiere scheint darin zu liegen, dass der Körper einem Steuerungssystem unterliegt, welches den Rhythmus von Organen und Zellen an Signale aus der Umwelt anpasst. Bei diesen Signalen kann es sich beispielsweise um Lichtreize handeln. Die Lichtreize werden über das Auge aufgenommen und letztendlich über den Sehnerv an die Epiphyse weiter geleitet. Die Epiphyse hat eine zentrale Funktion bei der Regulation der körpereigenen Rhythmen, die durch Zeitgeber dem Tagesrhythmus angepasst werden müssen. Die Signale werden von der Epiphyse an den mittleren Teil des Vorderhirns und das retikuläre Bindegewebe des Rückenmarks weitergeleitet. Von dort aus wird das Verhalten des Organismus beeinflusst (Fraser und Broom, 2004c).

Die Manipulation der Photoperiode wird als eine nichtinvasive, einfach zu realisierende Methode angesehen, um die Effektivität der Produktion zu verbessern. So konnten bspw. Bruininx et al. (2001) in Untersuchungen, in denen Ferkel zwölf Stunden am Tag bei künstlichem Licht und zwölf Stunden am Tag bei Dunkelheit gehalten wurden, feststellen, dass die erste Futteraufnahme nach dem Absetzen immer in der Lichtphase erfolgte. In weiteren Untersuchungen beobachteten Bruininx et al. (2002b), dass die durchschnittliche tägliche Futterverwertung in den ersten beiden 
Aufzuchtswochen von $16 \%$ auf $38 \%$ ansteigt, wenn der Beleuchtungszyklus von acht Stunden Licht auf 23 Stunden Licht erhöht wird. Hsia und Wood-Gush (1984) konnten keinen Einfluss einer verlängerten Lichtperiode auf das Futteraufnahmeverhalten und die Gewichtsentwicklung nachweisen. In Untersuchungen verglichen sie die Futteraufnahme bei Schweinen im Gewichtsbereich von $20 \mathrm{~kg}$ bis $80 \mathrm{~kg}$, die bei 24-stündiger Dauerbeleuchtung gehalten wurden mit der von Tieren, die einer 8,5-stündigen Photoperiode ausgesetzt waren. Tiere, die bei einer 8,5-stündigen Beleuchtungsdauer gehalten wurden, verbrachten während dieser Periode mehr Zeit mit der Futteraufnahme als die Tiere, die einer ständigen Beleuchtungsdauer ausgesetzt waren. Ein 24-stündiger Lichttag führte jedoch zu mehr Mahlzeiten am Tag.

\subsubsection{Mischen wurffremder Ferkel}

Unter natürlichen Bedingungen haben Ferkel etwa eine Woche nach der Geburt erste soziale Kontakte zu wurffremden Ferkeln und Sauen (Jensen und Redbo, 1987; Petersen et al., 1989). Mit zunehmendem Alter nimmt die Distanz zwischen den verschiedenen Würfen ab, bis die Phase der sozialen Integration abgeschlossen ist und die Ferkel vollständig in den Familienverband integriert sind (Jensen, 2002). In der Literatur liegen unterschiedliche Auffassungen darüber vor, wie die soziale Integration der Tiere sich vollzieht:

- Stufenlose Integration: Die Sau und ihre Ferkel gliedern sich der Herde vollständig an und nehmen sowohl tagsüber als auch nachts am Herdenleben teil (Stangel und Jensen, 1991).

- Stufenweise Integration: Die Sau und ihre Ferkel nehmen an den Tagesaktivitäten der Rotte teil, schlafen aber nachts abgesondert von der Herde (Dellmeier und Friend, 1991; Jensen und Redbo, 1987; Stangel und Jensen, 1991).

- Bildung zeitlicher Kommunen: Es kommt zur Bildung zeitlicher Kommunen zwischen mehreren führenden Tieren, bevor eine endgültige Eingliederung in die Rotte erfolgt (Newberry und Wood-Gush, 1986). 
In der konventionellen Schweinehaltung erfolgt das Absetzen der Ferkel i.d.R. nicht in einem stufenweisen Prozess, sondern stellt ein abruptes Ereignis dar. Die Ferkel werden in eine neue Umgebung umgestallt und durch das Mischen unterschiedlicher Würfe entstehen größere neue Gruppen (Brooks und Tsourgiannis, 2003). Die Konfrontation mit wurffremden Ferkeln stellt dabei erhebliche Anforderungen an die Adaptionsfähigkeit der Tiere. In den neu zusammen gesetzten Gruppen aus wurffremden Ferkeln werden die Tiere nach dem Absetzen gezwungen, eine neue Rangordnung zu etablieren. Das Mischen wurffremder Ferkel wird als Hauptursache für das Auftreten von Aggressionen angesehen (Keeling und Jensen, 2002; Weary et al., 1999).

In zahlreichen Untersuchungen konnte ein positiver Effekt des Mischens von wurffremden Ferkeln bereits in der Säugephase nachgewiesen werden (D'Eath, 2005; Ekkel et al., 1995; Pitts et al., 2005; Weary et al., 2007).

Durch das Mischen wurffremder Ferkel in der Säugephase, wird den Ferkeln die Möglichkeit gegeben, schon bei Anwesenheit der Sau eine neue Rangordnung zu etablieren. Dadurch kommt es in den ersten Aufzuchttagen zu weniger Aggressionen (D'Eath, 2005; Weary et al., 1999). Die Ferkel verbringen weniger Zeit mit der Etablierung einer neuen Rangordnung und mehr Zeit mit der Futteraufnahme (Algers et al., 1990; Wattanakul et al., 1997; Weary et al., 1999). Auch Li und Johnston (2009) konnten in neuesten Untersuchungen zeigen, dass Ferkel, die bereits vor dem Absetzen in Gruppen gehalten wurden, weniger aggressives Verhalten zeigten. Des Weiteren war die durchschnittliche Dauer der Kämpfe geringer, wenn wurffremde Ferkel bereits vor dem Absetzen sozialisiert wurden.

\subsection{Abgeleitete Vorgehensweisen}

Dem Prozess des Absetzens wird seit einigen Jahren sowohl in der Wissenschaft als auch in der praktischen Landwirtschaft immer mehr Bedeutung zugemessen (Pluske et al., 2003).

Der Absetzprozess ist mit einer Reihe von Stressoren verbunden, die in einer gesteigerten Stressantwort der Tiere auf das Absetzen resultieren. Die optimale Kombination von Einflussfaktoren wie Absetzalter, Gruppengröße, Futterkonsistenz und Futterbereitstellung, Tier-Fressplatzverhältnis, 
Photoperiode und das Mischen wurffremder Ferkel stellt den Schlüssel für eine wirtschaftlich erfolgreiche Schweinehaltung dar (Weary et al., 2007).

Im Fokus der Wissenschaft muss daher eine genaue Untersuchung der einzelnen Faktoren stehen, um die Bedeutung, die diese Faktoren auf die Entwicklung von abgesetzten Ferkeln haben zu evaluieren, und mögliche Interaktionen zwischen den Faktoren aufzudecken. In früheren Untersuchungen konnte nachgewiesen werden, dass die Stressantwort der Tiere durch eine Modifikation von haltungs- und prozesstechnischen Faktoren vor und nach dem Absetzen beeinflusst werden kann und somit die Tiergerechtheit und die Wirtschaftlichkeit der einzelnen Produktionsstufen verbessert werden kann. Letztendlich sind jedoch Modifikationen von Haltungsumwelten gefordert, die sowohl für die Tiere einen Vorteil bedeuten, aber auch praktikabel sind und sich in herkömmlichen Systemen leicht umsetzen lassen. Es muss sich hierbei um realistische Managementsysteme handeln (Weary et al., 2007).

In der vorliegenden Arbeit sollte durch eine Anpassung verschiedener, bisher nicht berücksichtigter Faktoren der Absetzstress von abgesetzten Ferkeln minimiert und somit das Futteraufnahmeverhalten und die daraus resultierende Gewichtsentwicklung der Tiere verbessert werden. In einem ersten Versuchsteil (s. Kapitel 3.1) sollte die Attraktivität des Futters durch die Temperierung des Futterbreis in der ersten Woche nach dem Absetzen gesteigert werden. Ziel des zweiten Versuchsteils war es, durch eine Verlängerung der Photoperiode in den ersten vier Tagen nach dem Absetzen die Orientierung am Futtertrog zu steigern und somit eine erhöhte Futteraufnahme zu realisieren (s. Kapitel 3.3). Durch eine frühe Sozialisierung der Ferkel bereits vor dem Absetzen sollte in einem dritten Versuchsteil eine Minimierung der Stressantwort auf die kritische Phase des Absetzens und eine daraus resultierende gesteigerte Wachstumsleistung realisiert werden (s. Kapitel 3.4).

Um die wissenschaftliche Arbeit zu erleichtern und so schneller zu belastbaren Ergebnissen zu kommen, müssen unter Anderem neue Wege der Tierbeobachtung gegangen werden. Auch für den wirtschaftenden Betrieb sind genaue Daten aus der laufenden Produktion ein wichtiges Mittel, um die Schweinehaltung zu optimieren und somit wettbewerbsfähig zu bleiben.

Im Rahmen der vorliegenden Untersuchungen wurde daher, parallel zur Analyse der verschiedenen Einflussfaktoren, mit Hilfe der 
Radiofrequenzidentifikation (RFID) eine innovative Technik entwickelt und geprüft, mit der es möglich ist, Ferkel simultan individuell am Rundtrog von Futterautomaten zu identifizieren. Diese Technik soll zukünftig aufwändige Videobeobachtungen ablösen, die bisher nötig waren, um den Einfluss verschiedenster Faktoren in Hinblick auf das Tierverhalten und die Tierleistung zu analysieren und miteinander zu vergleichen (s. Kapitel 3.2). 


\section{Beiträge mit Begutachtung}

3.1 The effect of heated mash on performance and feeding behavior of newly weaned piglets

Reiners, K., E. F. Hessel und H. F. A. Van den Weghe

Journal of Animal Science 2008, 86, 3600-3607 
3.2 Application of RFID technology using passive HF transponders for the individual identification of weaned piglets at the feed trough

Reiners, K., A. Hegger, E. F. Hessel, S. Böck, G. Wendl und H. F. A. Van den Weghe

Computers and Electronics in Agriculture 2009, 68, 178-184 
3.3 Influence of photoperiod on behavior and performance of newly weaned piglets with a focus on feeding behavior, feed consumption and growth

Reiners, K., E. F. Hessel, S. Sieling und H. F. A. Van den Weghe Journal of Swine Health and Production: eingereicht, September 200 
3.4 Socializing piglets before weaning: Effects on behavior of lactating sows, pre- and postweaning behavior, and performance of piglets

Hessel, E. F., K. Reiners und H. F A. Van den Weghe Journal of Animal Science 2006, 84, 2847-2855 


\section{Diskussion}

\subsection{Evaluierung prozesstechnischer Einflussfaktoren}

Ein wesentlicher Grundstein für eine erfolgreiche und damit wirtschaftlich effiziente Schweineproduktion wird bereits in der Ferkelaufzucht gelegt. Die Adaptionsfähigkeit der Ferkel wird beim Absetzen häufig überfordert (Morméde und Hay, 2003). Durch eine Anpassung von Haltungs- und Fütterungssystemen an die natürlichen Verhaltensweisen von Ferkeln werden die Ansprüche an die Anpassungsfähigkeit verringert, eine reduzierte Stressantwort der Tiere auf das Absetzen sowie eine daraus resultierende Steigerung der biologischen Leistungen sind die Folge (Weary et al., 2007). Besondere Bedeutung kommt hierbei der Futteraufnahme der Ferkel direkt nach dem Absetzen zu. In zahlreichen Untersuchungen konnte nachgewiesen werden, dass Ferkel Wachstumsdepressionen nach dem Absetzen über den gesamten Verlauf der Aufzucht und der darauf folgenden Mast nicht mehr kompensieren (King und Pluske, 2003).

In der vorliegenden Arbeit konnte nachgewiesen werden, dass es eine Reihe von Faktoren gibt, die die Futteraufnahme der Ferkel nach dem Absetzen und damit die Gewichtsentwicklung der Tiere in der Aufzucht und der darauf folgenden Mast positiv beeinflussen. In insgesamt drei Versuchsteilen wurden der Einfluss einer Temperierung des Futterbreis in der ersten Woche nach dem Absetzen, die Bedeutung einer verlängerten Photoperiode in der ersten vier Tagen nach dem Absetzen, sowie der Effekt einer Sozialisierung wurffremder Ferkel bereits vor dem Absetzen analysiert.

Eines der Hauptprobleme beim Absetzen von Ferkeln stellt die Futterumstellung dar. Während das Absetzen unter natürlichen Bedingungen mit einer stufenweisen Umstellung von flüssiger Sauenmilch auf festes Futter einhergeht, erfolgt die Futterumstellung in der praxisüblichen Ferkelerzeugung abrupt (Held und Mendl, 2001; Jensen, 2002; King und Pluske, 2003). Durch die Temperierung des Futterbreies in der ersten Woche nach dem Absetzen wurde in der vorliegenden Untersuchung der Versuch unternommen, den Übergang von flüssiger Sauenmilch auf festes Futter weniger abrupt zu gestalten (s. Kapitel 3.1). Zum einen war das angebotene Futter der Sauenmilch aufgrund 
seiner breiförmigen Konsistenz ähnlicher als trockenes Futter, zum anderen entsprach die Temperatur des Futters der der Sauenmilch.

Zum Einfluss von temperiertem Futterbrei auf das Futteraufnahmeverhalten und die Aufzuchtsleistung von Ferkeln liegen bisher keine vergleichbaren Untersuchungen vor. In der Kälber- und Lämmeraufzucht hingegen konnte in früheren Untersuchungen ein positiver Effekt von angewärmter Milch auf die Leistung der Tiere nachgewiesen werden. So kam beispielsweise Large (1965) bei einem Vergleich kalter und warmer Tränke bei Lämmern zu dem Ergebnis, dass kalte Milch langsamer aufgenommen und schlechter verwertet wird als warme. Bauer et al. (1971) und Burkart und Bauer (1973) konnten in ihren Untersuchungen zeigen, dass eine Kalttränke von Kälbern bei gleichem Tränkeverfahren zu einer schlechteren Milchaufnahme führt. Die Autoren gehen davon aus, dass die Tränketemperatur der Körpertemperatur entsprechen sollte. Nach Meinung der Autoren entspricht die Warmtränke am ehesten den natürlichen Bedingungen.

In früheren Untersuchungen wurde von einem positiven Einfluss von fermentiertem Futter auf die Wachstumsleistung von abgesetzten Ferkeln berichtet (Mikkelsen und Jensen, 1998; Scholten et al., 2002). Die Ursache für den positiven Einfluss von fermentiertem Futter auf die Wachstumsleistung der Ferkel wird in einer verbesserten Darmgesundheit gesehen. Es ist hinlänglich bekannt, dass es durch die reduzierte Futteraufnahme der Ferkel nach dem Absetzen zu einer Atrophie der Darmzotten bei gleichzeitiger Hyperplasie der Krypten kommt, die eine verminderte Absorption der aufgenommenen Nährstoffe zur Folge hat (Lallés et al., 2004; Pluske et al., 1997). Scholten et al. (2002) konnten in Untersuchungen zeigen, dass die Atrophie der Darmzotten bei Ferkeln, die mit fermentiertem Flüssigfutter gefüttert wurden, geringer ausfiel als bei Ferkeln, die unfermentiertes Futter erhielten. Canibe und Jensen (2003) konnten darüber hinaus im Gastrointestinaltrakt von Ferkeln, die fermentiertes Futter erhielten, eine geringere Anzahl von Enterobakterien feststellen als im Gastrointestinaltrakt von Ferkeln, die unfermentiertes Futter erhielten. Die Fermentation scheint somit die Darmumwelt zu beeinflussen und die Verdaulichkeit einiger Futterkomponenten zu verbessern (Liu et al., 2007).

In der vorliegenden Untersuchung wurden keine physiologischen Untersuchungen des Intestinaltraktes durchgeführt. Die Gewichtsentwicklungen 
der Ferkel lassen jedoch darauf schließen, dass es durch die gesteigerte Attraktivität des Futters und die daraus resultierende erhöhte Futteraufnahme nach dem Absetzen zu einer verbesserten Verwertung der Nährstoffe im Organismus kam. Mit Mehrzunahmen von 0,89 kg während der Aufzucht (49 Tage) und $3,98 \mathrm{~kg}$ über den gesamten Versuchszeitraum (139 Tage) hinweg, zeigten die Tiere, die in der ersten Aufzuchtswoche temperierten Futterbrei erhielten, signifikant höhere Zunahmen als die Tiere, deren Futterbrei in der ersten Aufzuchtswoche nicht angewärmt wurde. Diese Ergebnisse werden von Owen und Larson (1982) bestätigt, die in ähnlichen Untersuchungen bei Kälbern, die mit $32-38^{\circ} \mathrm{C}$ warmer Milch gefüttert wurden, höhere Zunahmen feststellen konnten als bei Kälbern, die kalte Milch $\left(2-7^{\circ} \mathrm{C}\right)$ erhielten. Während die Tiere, die warme Milch erhielten, in den ersten sechs Lebenswochen Zunahmen von $0,46 \mathrm{~kg}$ pro Tag hatten, konnten bei den Kälbern, die im gleichen Zeitraum kalte Milch erhielten, Tageszunahmen von nur 0,37 kg registriert werden.

Neben dem Einfluss des temperierten Futterbreies wurde der Effekt einer Verlängerung der Photoperiode in den ersten vier Tagen nach dem Absetzen analysiert (s. Kapitel 3.3). Die Beleuchtungsdauer für Ferkelaufzuchtställe ist in der Bundesrepublik Deutschland durch die TierschutzNutztierhaltungsverordnung (2006) geregelt. Die Verordnung stützt sich unter anderem auf die EU- Richtlinie 2001/93/EG (2001). Neu gebaute Ställe müssen über eine Einfallfläche für Tageslicht von mindestens drei Prozent der Stallgrundfläche verfügen, darüber hinaus muss im Aufenthaltsbereich der Tiere eine möglichst gleichmäßige Verteilung des Tageslichts erreicht werden. Ist dieses nicht der Fall, muss durch künstliche Beleuchtung sichergestellt sein, dass der Stall, dem Tagesrhythmus der Tiere entsprechend, mindestens acht Stunden täglich mit einer Beleuchtungsstärke von $80 \mathrm{~lx}$ beleuchtet wird.

In früheren Untersuchungen konnte durch eine Verlängerung der Photoperiode über die gesamte Aufzuchtperiode hinweg die Effektivität der Produktion verbessert werden (Bruininx et al., 2002b). Die Manipulation der Photoperiode wird von vielen Autoren als eine nichtinvasive, einfach zu realisierende Methode angesehen, um die Effektivität der Produktion zu verbessern. 
Gestützt auf die Ergebnisse dieser Untersuchungen wurde der Ferkelaufzuchtstall in der vorliegenden Untersuchung nicht, wie in der Tierschutz-Nutztierhaltungsverordnung (2006) gefordert, nur acht Stunden pro Tag, sondern 20 Stunden pro Tag beleuchtet. Im Gegensatz zu früheren Untersuchungen wurde die Photoperiode jedoch nicht über die gesamte Aufzuchtperiode hinweg, sondern nur in den ersten vier Tagen nach dem Absetzen verlängert. Es wurde angenommen, dass der Aktivitätsrhythmus, den die Ferkel in den ersten vier Tagen nach dem Absetzen ausbilden, auch nach der Umstellung von 20 Stunden auf acht Stunden Beleuchtung am fünften Tag nach dem Absetzen von den Tieren beibehalten wird.

Im Gegensatz zu der Temperierung des Futterbreis in der ersten Woche nach dem Absetzen, resultierte die Verlängerung der Photoperiode in den ersten vier Tagen nach dem Absetzen nicht in einer Steigerung der Futteraufnahme der Ferkel. Der Futterverbrauch war bei den Ferkeln, die in den ersten vier Tagen nach dem Absetzen 20 Stunden Beleuchtung ausgesetzt waren, in den ersten 24 Stunden erhöht. Über den gesamten siebenwöchigen Versuchszeitraum konnte jedoch, im Gegensatz zu der Temperierung des Futterbreis, kein Einfluss der Photoperiode auf den Futterverbrauch der Tiere festgestellt werden. Während die Temperierung des Futterbreis in der ersten Woche nach dem Absetzen über die gesamte Aufzuchtperiode hinweg einen positiven Einfluss auf die Gewichtsentwicklung der Tiere hatte, konnten die täglichen Zunahmen der Ferkel durch die Verlängerung der Photoperiode nicht verbessert werden. Die Ergebnisse zeigen, dass der Effekt einer verlängerten Photoperiode in den ersten vier Tagen nach dem Absetzen geringer ist als der Einfluss von temperiertem Futterbrei in der ersten Woche nach dem Absetzen. Die Darreichungsform des Futters hatte in der vorliegenden Untersuchung einen größeren Einfluss auf das Futteraufnahmeverhalten, den Futterverbrauch und die Wachstumsleistung der Ferkel als die Photoperiode.

Die Sozialisierung von wurffremden Ferkeln bereits vor dem Absetzen wird von vielen Autoren als eine der wirksamsten Maßnahmen angesehen, um den Absetzstress und damit die Futteraufnahme der Ferkel nach dem Absetzen zu steigern (D'Eath, 2005; Ekkel et al., 1995; Pitts et al., 2000; Weary et al., 2007). 
Diese Annahme konnte in der vorliegenden Untersuchung bestätigt werden. Ferkel, die bereits unter Anwesenheit der Sau mit wurffremden Ferkeln sozialisiert wurden, zeigten nach dem Absetzen signifikant weniger Aggressionen, eine höhere Futteraufnahme und höhere Gewichtszunahmen als Ferkel, die erst nach dem Absetzen Kontakt zu wurffremden Ferkeln hatten. Der Grund für den positiven Einfluss einer frühen Sozialisierung wird darin gesehen, dass der Absetzprozess stufenweise einhergeht. Während es unter praxisüblichen Bedingungen zu einem abrupten Ereignis kommt, in dem die Ferkel zeitgleich von der Mutter getrennt, mit neuen Wurfgeschwistern konfrontiert, in eine neue Haltungsumwelt umgestallt, und mit neuem Futter konfrontiert werden, wird dieser Prozess durch die Sozialisierung der Ferkel bereits vor dem Absetzen entzerrt. Die Ferkel durchleben einige der Stressoren, die mit dem Absetzen verbunden sind, bereits bei Anwesenheit der Sau (Wattanakul et al., 1997; Weary et al., 2007).

Weary et al. (2007) sehen nicht nur in der Sozialisierung wurffremder Ferkel, sondern auch in der damit einhergehenden partiellen Abwesenheit der Ferkel von der Sau bereits vor dem Absetzen Vorteile. Die Autoren gehen davon aus, dass die Ferkel durch die partielle Abwesenheit von der Sau bereits vor dem Absetzen mehr festes Futter aufnehmen. Es wird häufig diskutiert, dass Ferkel, die vor dem Absetzen mehr festes Futter und weniger Milch aufnehmen, weniger stark auf den Absetzstress reagieren.

In der Literatur liegen unterschiedliche Auffassungen über die Gestaltung von Systemen vor, in denen es möglich ist, Ferkel bereits vor dem Absetzen zu sozialisieren und den Wurf partiell von der Sau zu trennen, da diese Systeme in der Regel mehr Arbeit und einen zusätzlichen Managementaufwand bedeuten. Ein möglicher Ansatz ist darin zu sehen, die Haltungssysteme so zu gestalten, dass Sau und Ferkel sich zeitweise freiwillig voneinander trennen. Die natürliche Mutter-Kind-Beziehung impliziert, dass die Zeit, die die Sau in einem solchen System entfernt von inrem Wurf verbringen wird, im Laufe der Zeit ansteigt (Jensen, 2002). Wenn die Sau die Ferkel freiwillig verlassen kann (bspw. über einen Outdoor-Auslauf oder über ein zusätzliches Tor), werden die Ferkel mehr festes Futter konsumieren, um die reduzierte Milchaufnahme zu kompensieren (Cox und Cooper, 2001; Damm et al., 2003; Hötzel et al., 2004; Pajor et al., 2002). Der Erfolg dieser Haltungssysteme hängt jedoch davon ab, 
in welchem Maße die Sau ihre Säugungen reduziert. In einigen Untersuchungen konnte beobachtet werden, dass die Sau ihre Säugungen nach vier Wochen Säugezeit komplett einstellte, in anderen Untersuchungen konnte nur eine sehr geringe Abnahme der Säugungen beobachtet werden (Pitts et al., 2000). Systeme, bei denen die Sau ihren Wurf freiwillig verlassen kann, haben neben einer erhöhten Aufnahme von festem Futter durch die Ferkel den Vorteil, dass die Ferkel sich langsam an die Abwesenheit der Sau gewöhnen (Weary et al., 2007). Es bleibt jedoch zu bedenken, dass eine Sozialisierung wurffremder Ferkel in derartigen Systemen nicht möglich ist.

Vor dem Hintergrund des Arbeits- und Managementaufwandes wurde in der vorliegenden Untersuchung ein System gewählt, in dem die Ferkel zwar bereits vor dem Absetzen die Möglichkeit hatten, sich mit wurffremden Ferkeln zu sozialisieren, die Sauen jedoch über die gesamte Säugezeit im Kastenstand fixiert blieben. Weary et al. (1999) sehen Systeme, wie sie in der vorliegenden Untersuchung gewählt wurden, als kritisch an. Die Ferkel verbringen zwar zwischen den Säugungen Zeit entfernt von der Sau, die Frequenz der Säugungen und die Menge der Milchaufnahme nehmen jedoch nicht ab, da die Ferkel jederzeit zur Sau zurück kehren können, um zu säugen. Die Festfutteraufnahme der Ferkel wurde in der vorliegenden Untersuchung nicht analysiert, es konnte jedoch bestätigt werden, dass die Frequenz der Säugungen nicht abnahm.

Auch bei anderen Tierarten brachte eine erzwungene Trennung von Mutter und Kind während der Säugephase Vorteile mit sich. Orgeur et al. (1998) beschreiben, dass Lämmer, die bereits ab der dritten Lebenswoche für vier bis acht Stunden täglich von ihrer Mutter getrennt wurden, beim Absetzen im Alter von vier Monaten sehr viel weniger Stress zeigten als Lämmer, die vor dem Absetzen durchgängig Kontakt $z u$ ihrer Mutter hatten. In der Pferdehaltung konnte nachgewiesen werden, dass Fohlen, die in Gruppen abgesetzt wurden, weniger Stress zeigten als Tiere, die nach dem Absetzen getrennt gehalten wurden (Heleski et al., 2002; Hoffmann et al., 1995). Auch bei Kälbern waren die Leistungen nach dem Absetzen verbessert, wenn die Kälber in Paaren zu zweit statt alleine abgesetzt wurden (Chua et al., 2002). Die Autoren gehen davon aus, dass die Tiere durch das paarweise Absetzen nur partiell auf die Isolation von der Mutter reagieren. 
Bei der Interpretation der Ergebnisse der vorliegenden Untersuchung bleibt zu bedenken, dass in den einzelnen Versuchsteilen jeweils nur ein möglicher Einflussfaktor verändert wurde. Es bleibt zu überprüfen, ob eine Kombination mehrerer Einflussfaktoren einen zusätzlichen Effekt bringt, oder ob die Einflussfaktoren sich gegenseitig aufheben. Weary et al. (2007) konnten zeigen, dass der Absetzstress durch die Nutzung mehrerer positiver Einflussfaktoren deutlich vermindert werden kann. Vor dem Hintergrund der vorliegenden Ergebnisse wäre es daher beispielsweise denkbar, Ferkeln, die bereits vor dem Absetzen sozialisiert wurden, in weiterführenden Untersuchungen nach dem Absetzen temperierten Futterbrei anzubieten. Die Auswahl der verschiedenen Kombinationsmöglichkeiten sollte jedoch neben der Effektivität auch vor dem Hintergrund der praktischen Umsetzbarkeit und des Management- und Kostenaufwandes getroffen werden.

\subsection{Entwicklung einer simultanen Einzeltiererkennung}

In der vorliegenden Untersuchung wurde die Evaluierung der verschiedenen Einflussfaktoren auf das Verhalten der Ferkel mit Hilfe aufwendiger Videobeobachtungen durchgeführt. Um Haltungs- und Fütterungssysteme für abgesetzte Ferkel zukünftig vor dem Hintergrund der Tiergerechtheit und damit der Effizienz der Ferkelaufzucht optimal gestalten zu können, sind zahlreiche weitere Verhaltensbeobachtungen nötig.

Der Arbeits- und damit auch Kostenaufwand, der mit einer Bewertung von Haltungs- und Fütterungssystemen verbunden ist, ist sehr hoch. Um diesen Aufwand zukünftig senken zu können, und gleichzeitig die Genauigkeit der Verhaltensbeobachtungen zu steigern, wurde in der vorliegenden Untersuchung parallel zur Evaluierung der haltungs- und prozesstechnischen Einflussfaktoren eine auf Radiofrequenzidentifikation (RFID) basierende simultane Einzeltiererkennung für Aufzuchtferkel unter Verwendung von High-FrequencyTranspondern (HF-Transpondern) an einem Futterautomaten mit mehreren Fressplätzen entwickelt und evaluiert (s. Kapitel 3.2).

Im Bereich der Tierkennzeichnung werden üblicherweise Low-FrequencyTransponder (LF-Transponder) eingesetzt, die sich nach den internationalen Standards ISO 11784 (1996a) und ISO 11785 (1996b) richten. Diese 
Technologie ist gut erprobt und findet bereits seit über 20 Jahren vielseitigen Einsatz in der Tierhaltung (Abrufstationen für Sauen und Kühe, automatische Melksysteme, Kälbertränkeautomaten). Der Nachteil der LF-Transponder besteht jedoch darin, dass sie im Empfangsbereich nur einzeln ausgelesen werden können (Kern, 2006). Mit Hilfe von LF-Tranpondern wurden in früheren Untersuchungen bereits elektronische Futterautomaten eingesetzt, um die individuelle Futteraufnahme, die Anzahl und die Dauer der Trogbesuche zu registrieren (Georgsson und Svendsen, 2002; Hyun et al., 1997). Bei der elektronischen Erfassung der Tiere traten jedoch zum einen sehr viele Fehler auf (De Haer et al., 1992; Slader und Gregory, 1988), zum anderen war die Registrierung der Tiere nur möglich, wenn die Automaten so gestaltet waren, dass nur ein Tier zur Zeit fressen kann (Hyun et al., 1997). Derartige Automaten werden heutzutage nur noch vereinzelt in Zuchtbetrieben oder in Forschungseinrichtungen genutzt und sind zur Bewertung des Fressverhaltens von abgesetzten Ferkeln an praxisüblichen Rundtrögen nicht geeignet.

Bei der Bewertung des Fressverhaltens von abgesetzten Ferkeln ist es erforderlich, dass die Ferkel simultan individuell am Rundtrog der Futterautomaten erkannt werden können. Diese Forderung kann unter bestimmten Voraussetzungen, die in Kapitel 3.2 ausführlich diskutiert wurden, mit Hilfe der in der vorliegenden Untersuchung entwickelten Transpondertechnologie erfüllt werden.

Vor dem Hintergrund stark wachsender Herdengrößen, erhöhter Arbeits- und Verwaltungsanforderungen, gesetzlicher Auflagen sowie einer damit einher gehenden steigenden Datenflut wäre es denkbar, das entwickelte RFID-System nicht nur für Forschungszwecke, sondern auch für die innerbetriebliche Prozesskontrolle und überbetriebliche Kontrollsysteme zu nutzen.

Landwirtschaftliche Betriebe sind durch die Aufzeichnungspflichten, die wachsenden Bestandesgrößen und die zunehmende Automatisierung der Betriebe mit einer immer größer werdenden Datenflut konfrontiert. Der Datentransfer zwischen Betrieben, Händlern, Schlachthöfen und der Tierverkehrsdatenbank nimmt insbesondere vor dem Hintergrund der Forderung nach einer durchgängigen Rückverfolgbarkeit zu. Unter dem Stichwort der Lebensmittelsicherheit und Lebensmittelqualität besteht auch von 
Seiten des Konsumenten eine Forderung nach Rückverfolgbarkeit der Produkte durch die gesamte Produktions- und Verarbeitungskette.

In den letzten 10 Jahren wurde die Handhabung und die Funktionsfähigkeit verschiedener elektronischer Kennzeichnungssysteme untersucht: Elektronische Ohrmarken, Boli und Injektate. Die Untersuchungen ergaben, dass die elektronische Ohrmarke Vorteile bei der Applikation im landwirtschaftlichen Betrieb und auch bei der späteren Entnahme im Schlachthof hat (Klindtworth, 1998; Klindtworth et al., 2002; Zähner und Spiessl-Mayer, 2005). Bisher wurden zum Zweck der elektronischen Tierkennzeichnung LF-Transponder eingesetzt. LF-Transponder sind nicht kollisionsfähig, die Tiere müssen daher zum Lesen der Transponder vereinzelt werden (Finkenzeller, 2002). Aus arbeitswirtschaftlichen und produktionstechnischen Gründen wäre es jedoch sinnvoll und erforderlich, dass Tiere simultan identifiziert werden können (beispielsweise bei der Verladung oder am Schlachthof). Durch eine Weiterentwicklung des in der vorliegenden Untersuchung entwickelten RFID-Systems wäre es denkbar, die Tiere mit Hilfe von HF-Transpondern unter Einsatz eines Antikollisionssystems so zu kennzeichnen, dass sie simultan registriert werden können. Bei der Kennzeichnung der Tiere mit HF-Transpondern wäre eine einfache und sichere Entnahme am Schlachthof gewährleistet. Die Transponder können nach der Geburt bei Applikation der Betriebsohrmarke auf den Dornteil der Ohrmarke aufgesteckt werden und am Schlachthof gemeinsam mit der Ohrmarke ohne Probleme entnommen werden. Die Gefahr, dass Transponder unbeabsichtigt in die Nahrungskette gelangen (Webb, 2004) wäre nicht gegeben. Bei einem Einsatz von HF-Transpondern muss jedoch beachtet werden, dass der Verbindungsweg zwischen Transponder und Antenne bei hoher Frequentierung eingeschränkt ist, da der hohe Wassergehalt im Körper die Funkübertragung negativ beeinflusst (Kern, 2006). Bei der Gestaltung der verwendeten Antennen sollte dieses berücksichtigt werden.

Die elektronische Kennzeichnung lässt die telemetrische Datensammlung für die Qualitätskontrolle in der Schweineproduktion zu (Madec et al., 2001) und wird für die zukünftige Sicherung von Nahrungsmitteln eine große Rolle spielen (Intrakamhaeng et al., 2007). Durch RFID befindet sich die Identifikation von 
Nutztieren im Wandel. Neben der Tatsache, dass RFID für nationale und vom Gesetzgeber geforderte Identifikationssysteme eine praktikable Lösung bietet, ist in RFID großes strategisches Potential, auch für die Steuerung innerbetrieblicher Prozesse zu erkennen. RFID kann als Schlüsseltechnologie für das Precision Livestock Farming angesehen werden (Trevarthen, 2007). 


\section{$5 \quad$ Zusammenfassung}

Die Höhe der Gewichtszunahmen in der ersten Woche nach dem Absetzen stellt einen der wichtigsten Einflussfaktoren auf die Gewichtsentwicklung von Ferkeln bis zur Schlachtung dar. Unter optimalen Bedingungen haben frisch abgesetzte Ferkel ein sehr hohes Wachstumspotential. Dieses wird in der Regel jedoch nicht ausgeschöpft, da die Tiere beim Absetzen einer Reihe von Stressoren ausgesetzt werden, die das Futteraufnahmeverhalten und somit die Gewichtsentwicklung der Ferkel negativ beeinflussen.

In der vorliegenden Arbeit wurde der Einfluss verschiedener haltungs- und prozesstechnischer Faktoren auf die Wachstumsleistung und das Verhalten von abgesetzten Ferkeln evaluiert. In vergleichenden Untersuchungen wurde zum einen der Effekt des Mischens wurffremder Ferkel bereits vor dem Absetzen untersucht, zum anderen wurde der Einfluss eines temperierten Futterbreis und einer verlängerten Photoperiode nach dem Absetzen analysiert. Ziel der unterschiedlichen Versuchsansätze war es, den Absetzstress der Ferkel durch eine Optimierung der Haltungsumwelt und damit einer Reduktion von verschiedenen Stressoren zu minimieren, und die Futteraufnahme sowie die daraus resultierende Wachstumsleistung der Ferkel nach dem Absetzen zu steigern.

Durch das Mischen wurffremder Ferkel bereits vor dem Absetzen konnte der Anteil aggressiver Auseinandersetzungen nach dem Absetzen deutlich reduziert werden $(P<0,001)$. Einhergehend mit den verminderten aggressiven Auseinandersetzungen hatte das frühzeitige Mischen der Ferkel einen signifikanten Einfluss auf die täglichen Zunahmen der Tiere nach dem Absetzen. Über den gesamten Beobachtungszeitraum hinweg zeigten die Ferkel, die bereits in Anwesenheit der Sau mir wurffremden Ferkeln sozialisiert wurden, Mehrzunahmen von 1,09 $\pm 0,31 \mathrm{~kg}(P=0,049)$.

Auch die Temperierung des Futterbreis in der ersten Woche nach dem Absetzen hatte einen positiven Effekt auf die Wachstumsleistung der Ferkel. Während einer 49-tägigen Aufzuchtphase waren die Gewichtszunahmen der Ferkel, die in der ersten Woche nach dem Absetzen temperierten Futterbrei erhielten, $0,89 \pm 0,23 \mathrm{~kg}$ höher als die Zunahmen der Ferkel in der 
Referenzgruppe $(P=0,03)$. Die Ferkel der Versuchgruppe konsumierten über die gesamte Aufzuchtphase hinweg 42,56 $\pm 0,15 \mathrm{~kg}$ Futter, wohingegen die Tiere der Referenzgruppe in gleichen Zeitraum nur 37,15 $\pm 0,15 \mathrm{~kg}$ Futter aufnahmen $(P=0,023)$.

Die Verlängerung der Photoperiode von acht Stunden auf 20 Stunden in den ersten vier Tagen nach dem Absetzen resultierte in einem tendenziell höheren Futterverbrauch der Ferkel in den ersten 24 Stunden nach dem Absetzen $(P=0,087)$. Über die gesamte siebenwöchige Aufzuchtperiode hinweg konnte der Futterverbrauch der Ferkel nicht gesteigert werden. Die Gewichtsentwicklung und das Futteraufnahmeverhalten der Ferkel wurden von einer Verlägerung der Photoperiode in den ersten vier Tagen nach dem Absetzen nicht beeinflusst.

In den vorliegenden Untersuchungen konnte bestätigt werden, dass es eine Reihe von Einflussfaktoren gibt, die das Futteraufnahmeverhalten und die Wachstumsleistung von abgesetzten Ferkeln beeinflussen. Die Ergebnisse zeigen, dass in der Gestaltung der Haltungsumwelt und der Fütterungssysteme Potentiale liegen, die zukünftig genutzt werden sollten, um die Ferkelproduktion vor dem Hintergrund der Tiergerechtheit und der Wirtschaftlichkeit zu optimieren. In weiterführenden Untersuchungen bleibt zu überprüfen, ob durch eine Kombination der verschiedenen untersuchten Einflussfaktoren ein zusätzlicher positiver Effekt auf das Futteraufnahmeverhalten und die Wachstumsleistung der Ferkel realisiert werden kann.

Die Evaluierung von verschiedenen Einflussfaktoren auf das Verhalten von abgesetzten Ferkeln ist bisher nur mit Hilfe aufwendiger Videobeobachtungen möglich. In der vorliegenden Untersuchung wurde daher parallel zur Evaluierung der haltungs- und prozesstechnischen Einflussfaktoren eine auf Radiofrequenzidentifikation (RFID) basierende simultane Einzeltiererkennung für Aufzuchtferkel unter Verwendung von High- Frequency (HF)- Transpondern an einem Futterautomaten mit mehreren Fressplätzen entwickelt und geprüft. Die Erkennungsrate der simultanen Einzeltiererkennung war mit 97,3\% sehr hoch. Das System stellt eine gute Möglichkeit dar, Ferkel am Rundtrog von Futterautomaten online simultan zu identifizieren. 
Durch den Einsatz einer simultanen Einzeltiererkennung können Fütterungssysteme und Haltungsumwelten für Ferkel zukünftig effizienter evaluiert werden. Vor dem Hintergrund stark wachsender Herdengrößen, erhöhter Arbeits- und Verwaltungsanforderungen, gesetzlicher Auflagen sowie einer damit einher gehenden steigenden Datenflut wäre es des Weiteren denkbar, das entwickelte RFID-System sowohl für die innerbetriebliche Prozesskontrolle als auch für überbetriebliche Kontrollsysteme zu nutzen. Aufgrund der stetig steigenden Automatisierung im Bereich der Prozesssteuerung gelten RFID-Systeme als Schlüsseltechnologie und werden in der Nutztierhaltung zukünftig weiter an Bedeutung gewinnen. 


\section{Summary}

The amount of weight gain in the first weeks after weaning is an important factor on the weight development of piglets until the time of slaughter. Under ideal conditions the growth potential of newly weaned piglets is very high. However, the growth potential is often not fully exploited as animals are exposed to a number of stressors in the weaning process which have a negative effect on feeding behaviour and with that on weight gain.

The effect of various housing and process-related factors on growth performance and behaviour of weaned piglets was evaluated in the present study. Comparative studies were carried out to investigate the effect of mixing piglets from different litters prior to weaning, and also to analyse the influence of heated mash and prolonged photoperiod after weaning. The objective of the different experimental approaches was to reduce weaning stress for piglets to a minimum by optimising the housing environment and with that reduce various stressors, and to increase food uptake as well as the resulting growth performance of piglets following weaning.

By mixing piglets from different litters prior to weaning, the ratio of aggressive incidents after weaning could be significantly reduced $(P<0,001)$. In keeping with the reduction of aggressive incidents, mixing of litters had a significant impact on daily weight gain of animals post-weaning. Piglets, which had been socialised with piglets from other litters before separation from the sow, showed higher weight gains of $1,09 \pm 0,31 \mathrm{~kg}(P=0,049)$ over the entire observation period.

Providing heated mash in the first week after weaning also had a positive effect on the growth performance of piglets. Overall, during the 49-day rearing period the weight gain in piglets, which had received heated mash in the first week after weaning, was by $0,89 \pm 0,23 \mathrm{~kg}$ higher than the weight gain in piglets of the reference group $(P=0,03)$. Piglets in the experimental group consumed $42,56 \pm 0,15 \mathrm{~kg}$ of feed over the entire rearing period, whereas piglets of the reference group only consumed $37,15 \pm 0,15 \mathrm{~kg}$ of feed $(P=0,023)$ over the same period. 
An extension of the photoperiod from eight hours to 20 hours during the first four days after weaning resulted in tendentially higher feed consumption by piglets in the first 24 hours after weaning $(P=0,087)$. However, over the entire seven week rearing period feed consumption could not be increased by prolonging the photoperiod. Furthermore, the prolongation of the photoperiod in the first four days after weaning did not influence weight gain and feeding behavior of the piglets.

The present studies demonstrated that there are a number of factors which have an effect on feeding behaviour and growth performance of weaned piglets. Results show that potential lies in the organisation of housing environment and feeding systems. This potential is to be used in the future to optimise piglet production against the backdrop of animal welfare and profitability. A possible positive effect of a combination of the different factors which were analyzed in the present study should be verified in further studies.

Until now the evaluation of different factors which have an impact on the behaviour of weaned piglets has only been possible with the aid of video observations. Parallel to the evaluation of housing and process-related factors, simultaneous individual animal identification in rearing piglets based on radio frequency identification (RFID) using high frequency (HF) transponders at an automatic feeder with several feeding places has been developed and tested in the present study. With $97,3 \%$ the identification rate of simultaneous individual animal identification was very high. The system presents a good method to identify piglets simultaneously online at the round trough of an automatic feeder. Housing and process-related factors which have an impact on the behaviour of weaned piglets can be evaluated better in the future by using simultaneous individual animal identification at the automatic feeder. In the context of growing herd-sizes, increased work- and management requirements, legal restraints as well as soaring data volumes, it would be thinkable that the developed RFID system could be used in company internal process management as well as in management systems at intercompany level. RFID-systems are considered as key technology in precision livestock farming and will become increasingly important in the future. 


\section{$7 \quad$ Literaturverzeichnis}

Achebe, C. (1975): Vergleichende Untersuchungen einiger Verhaltensweisen von konventionellen und früh abgesetzten Ferkeln unter Berücksichtigung des Tierschutzes. Dissertation, Humboldt-Universität Berlin.

Algers, B., P. Jensen, und L. Steinwall (1990): Behaviour and weight changes at weaning and regrouping of pigs in relation to teat quality. Applied Animal Behaviour Science 26, 143-155.

Bauer, J., M. Kirchgessner, und M. Burgkart (1971): Zur mutterlosen Lämmeraufzucht. Züchtungskunde 43, 55-62.

Bigelow, J. A., und T. R. Houpt (1988): Feeding and drinking patterns in young pigs. Physiology and Behavior 43, 99-109.

Braun, S., und D. Marx (1994): Verhalten von Schweinen während der Aufzucht und der Mast in einem Haltungssystem mit Ruhekisten. In: KTBL (Hrsg.): Aktuelle Arbeiten zur artgemäßen Tierhaltung. KTBL-Schrift 356, Darmstadt, 190-192. ISBN: 3-7843-1909-2.

Brooks, P. H., und C. A. Tsourgiannis (2003): Factors affecting the voluntary feed intake of the weaned pig. In: J. R. Pluske, J. Le Dividich, und M. W. A. Verstegen (Hrsg.): Weaning the pig - concepts and consequences. Wageningen Academic Publishers, Wageningen, The Netherlands, 81-115. ISBN: 9-0768-9817-5.

Bruininx, E. M. A. M., C. M. C. van der Peet-Schwering, J. W. Schrama, P. F. G. Vereijken, P. C. Vesseur, H. Everts, L. A. den Hartog, und A. C. Beynen (2001): Individually measured feed intake characteristics and growth performance of group-housed weanling pigs: Effects of sex, initial body weight, and body weight distribution within groups. Journal of Animal Science 79, 301-308.

Bruininx, E. M. A. M., G. P. Binnendiyk, C. M. C. van der Peet-Schwering, J. W. Schrama, L. A. den Hartog, H. Everts, und A. C. Beynen (2002a): Effect of creep feed consumption on individual feed intake characteristics and performance of group-housed weanling pigs. Journal of Animal Science $80,1413-1418$.

Bruininx, E. M. A. M., M. J. W. Heetkamp, D. van den Bogaart, C. M. C. van der Peet-Schwering, A. C. Beynen, H. Everts, L. A. den Hartog, und J. W. Schrama (2002b): A prolonged photoperiod improves feed intake and energy metabolism of weanling piglets. Journal of Animal Science 80,1736-1745.

Buchholz, M. (1990): Das Verhalten früh abgesetzter Ferkel in Wahlversuchen bei unterschiedlichen Flächengrößen auf planbefestigtem Boden mit Einstreu oder Tiefstreu. Disstertation, Universität Hohenheim. 
Burgkart, M., und J. Bauer (1973): Das Produktionsverfahren mutterlose Aufzucht - intensive Lämmermast. In: M. Burkhart, J. Bauer, und F. Raue (Hrsg.): Mutterlose Lämmeraufzucht - intensive Lämmeraufzucht. DLG-Verlags-GmbH, Frankfurt am Main, 136. ISBN: 3-7690-3077-X.

Canibe, N., und B. B. Jensen (2003): Fermented and nonfermented liquid feed to growing pigs: Effect on aspects of gastrointestinal ecology and growth performance. Journal of Animal Science 81, 2019-2031.

Chua, B., E. Coenen, J. van Delen, und D. M. Weary (2002): Effects of pair versus individual housing on the behavior and performance of dairy calves. Journal of Dairy Science 85, 360-364.

Colson, V., P. Orgeur, A. Foury, und P. Morméde (2006): Consequences of weaning piglets at 21 and 28 days on growth, behaviour and homonal responses. Applied Animal Behaviour Science 98, 70-88.

Cox, L., und J. Cooper (2001): Observations on the pre- and postweaning behaviour of piglets reared in a commercial indoor and outdoor environment. Animal Science 72, 75-86.

Damm, B. I., L. J. Pedersen, L. B. Jessen, S. M. Thamsborg, H. Mejer, und A. K. Ersboll (2003): The gradual weaning process in outdoor sows and piglets in relation to nematode infections. Applied Animal Behaviour Science 82, 101-120.

D'Eath, R. B. (2005): Socialising piglets before weaning improves social hierarchy formation when pigs are co-mingled post-weaning. Applied Animal Behaviour Science 93, 199-211.

De Haer, L. C. M., J. W. M. Merks, H. G. Kooper, G. A. J. Buiting, und J. A. van Hattum (1992). A note on the IVOG-stations: A station to record the individual food intake of group-housed growing pigs. Animal Production 54, 160-162.

Dellmeier, G. R., und T. H. Friend (1991): Behaviour and extensive management of domestic sows (Sus Scrofa) and litters. Applied Animal Behaviour Science 29, 327-341.

Dritz, S. S., K. Q. Owen, J. L. Nelssen, R. D. Goodband, und M. D. Tokach (1996): Influence of weaning age and nursery diet complexity on growth performance and carcass characteristics and composition of high-health status pigs from weaning to 109 kilograms. Journal of Animal Science 74, 2975-2984.

Duven, K. (1991): Das Verhalten früh abgesetzter Ferkel in zweifaktoriellen Wahlversuchen. Dissertation, Universität Hohenheim.

Ekkel, E. D., C. E. A. van Doorn, M. J. C. Hessing, and M. J. M. Tielen (1995): The specific-stress-free housing system has positive effects on productivity, health and welfare of pigs. Journal of Animal Science 73, 1544-1551. 
Finkenzeller, K. (2006): RFID-Handbuch: Grundlagen und praktische Anwendungen induktiver Funkanlagen, Transponder und kontaktloser Chipkarten. 4. Auflage, Hanser, München. ISBN: 3-4464-0398-1.

Fraser, A. F., und D. M. Broom (1996a): Abnormal Behaviour 1, Stereotypien. In: A. F. Fraser, und D. M. Broom (Hrsg.): Farm animal behaviour and welfare. CAB International, Wallingford, Oxon, UK, 305-317. ISBN: 08519-9160-2.

Fraser, A. F., und D. M. Broom (1996b): Feeding. In: A. F. Fraser, und D. M. Broom (Hrsg.): Farm animal behaviour and welfare. CAB International, Wallingford, UK, 79-98. ISBN: 0-8519-9160-2.

Fraser, A. F., und D. M. Broom (1996c): Organization of behaviour in the individual animal. In: A. F. Fraser, und D. M. Broom (Hrsg.): Farm animal behaviour and welfare. CAB International, Wallingford, Oxon, UK, 69135. ISBN: 0-8519-9160-2.

Georgsson, L., und J. Svendsen (2002): Degree of competition at feeding differentially affects behavior and performance of group-housed growingfinishing pigs of different relative weights. Journal of Animal Science 80, 376-383.

Gonyou, H. W., E. Baltranena, D. Whittington, und J. Patience (1998): The behaviour of pigs weaned at 12 and 21 days of age from weaning to market. Canadian Journal of Animal Science 78, 517-523.

Gonyou, H. W. (1999): Feeder and pen design to increase efficiency. Advances in Pork Production 10, 103.

Gonyou, H. W., (2001): The social behaviour in pigs. In: L. J. Keeling, und H. W. Gonyou (Hrsg.): Social behaviour in farm animals. CAB International, Wallingford, Oxon, UK, 147-168. ISBN: 0-8519-9397-4.

Grauvogel, A. (1970): Angewandte Ethologie beim Hausschwein. Deutsche Tierärztliche Wochenschrift 77, 126-132.

Hampson, D. J. (1986): Attempts to modify changes in the piglet small intestine after weaning. Research in Veterinary Science 40, 313-317.

Hedemann, M. S., S. Højsgaard, und B. B. Jensen (2003): Small intestinal morphology and activity of intestinal peptidases in piglets around weaning. Journal of Animal Physiology and Animal Nutrition 87, 32-41.

Held, S., M. Mendl (2001): Behaviour of the young weaned pig. In: M. A. Varley und J. Wiseman (Hrsg.): The weaner pig - nutrition and management. CAB International, Wallingford, Oxon, UK, 273-297. ISBN: 0-85199532-2. 
Heleski, C. R., A. C. Shelle, B. D. Nielsen, und A. J. Zanella (2002): Influence of housing on weanling horse behavior and subsequent welfare. Applied Animal Behaviour Science 78, 291-302.

Hessel, E. F., und H. F. A. Van den Weghe (2005): Feed level controlled mash feeder vs. conventional tube mash feeder: Performance and eating behaviour of young weaned piglets. In: S. Cox (Hrsg.): Precision Livestock Farming '05. Wageningen Academic Publishers, Wageningen, The Netherlands, 211-218. ISBN: 9-0769-9868-X.

Hillmann, E., F. von Hollen, B. Bünger, und L. Schrader (2001): Einfluss von Gruppen- und Einzelabferkelung auf das Verhalten von Saug- und Absetzferkeln. In: KTBL (Hrsg.): Aktuelle Arbeiten zur artgemäßen Tierhaltung. KTBL-Schrift 403, Darmstadt, 37-45. ISBN: 3-7843-2132-1.

Hoffmann, R. M., D. S. Kronfield, J. L. Holland, und K. Greiwe-Crandell (1995): Preweaning diet and stall weaning method influences on stress response in foals. Journal of Animal Science 73, 2922.

Hötzel, M. J., L. C. Pinheiro Machacho, F. M. Wolf, und O. A. Dalla Costa (2004): Behaviour of sows and piglets reared in intensive outdoor or indoor systems. Applied Animal Behaviour Science 86, 27-39.

Hsia, L. C., und D. G. M. Wood-Gush (1984): Social facilitation in the feeding behaviour of pigs and the effect of rank. Applied Animal Etholology 11, 265-270.

Hyun, Y., M. Ellis, F. K. McKeith, und E. R. Wilson (1997): Feed intake pattern of group-housed growing-finishing pigs monitored using a computerized feed intake recording system. Journal of Animal Science 75, 1443-1451.

Ingram, D. L., D. E. Walters, und K. F. Legge (1980): Variations in motor activity and in food and water intake over $24 \mathrm{~h}$ periods in pigs. Journal of Agricultural Science 95, 371-380.

Intrakamhaeng, M., V. Chavananikul, und A. Suriyasomboon (2007): Financial feasibility study of Radio Frequency Identification (RFID) implementation in pig and dairy Farms. Proceedings of the Chulalongkorn University Veterinary Science Annual Congress, 26.-27. April 2007, 86.

ISO (1996a): ISO 11784. Radio Frequency Identification of animals - Code Structure. International Organisation for Standardisation.

ISO (1996b): ISO 11785. Radio Frequency Identification of Animals - Technical Concept. International Organisation for Standardisation.

Jensen, P., I. Redbo (1987): Behaviour during nest leaving in free-ranging domestic pigs. Applied Animal Behaviour Science 18, 355-362.

Jensen, P., B. Recén (1989): When to wean - observations from free-ranging domestic pigs. Applied Animal Behaviour Science 23, 49-60. 
Jensen, P., G. Stangel (1992): Behaviour of piglets during weaning in a seminatural enclosure. Applied Animal Behaviour Science 23, 227-238.

Jensen, P. (1994): Fighting between unacquainted pigs - effects of age and of individual reaction pattern. Applied Animal Behaviour Science 41, 37-52.

Jensen, P. (1995): The weaning process of free-ranging domestic pigs: Withinand between-litter variations. Ethology 100, 14-25.

Jensen, P. (2002): Behaviour of pigs. In: P. Jensen (Hrsg.): The ethology of domestic animals - an introductory text. CAB International, Wallingford, UK, 159-172. ISBN: 0-8519-9602-7.

Jeroch, H., W. Drochner, und O. Simon (1999): Fütterung der Schweine. In: Ernährung landwirtschaftlicher Nutztiere. Verlag Eugen Ulmer, Stuttgart, 319-336. ISBN: 3-8252-8180-9.

Keeling, L., und P. Jensen (2002): Behavioural disturbances, stress and welfare. In: P. Jensen (Hrsg.): The ethology of domestic animals - an introductory text. $\mathrm{CAB}$ International, Wallingford, UK, 79-98. ISBN: 0.8519-9602-7.

Kelly, D., und A. G. P. Coutts (2000): Development of digestive and immunological function in neonates: Role of early nutrition. Livestock Production Science 66, 161-167.

Kern, C. (2006): Anwendung von RFID-Systemen. 2. Auflage, Springer-Verlag, Berlin, Heidelberg. ISBN: 3-5404-4477-7.

Kim, J. H., K. N. Heo, J. Odle, I. K Han, und R. J. Harrell (2001): Liquid diets accelerate the growth of early-weaned pigs an the effects are maintained to marked weight. Journal of Animal Science 79, 427-434.

King, R. H., und J. R. Pluske (2003): Nutritional management of the pig in preperation of weaning. In: J. R. Pluske, J. Le Dividich und M. W. A. Verstegen (Hrsg.): Weaning the pig-concepts and consequences. Wageningen Academic Publishers, Wageningen, The Netherlands, 3751. ISBN: 9-0769-9817-5.

Kircher, A. (2001): Untersuchungen zum Tier-Fressplatzverhältnis bei der Fütterung von Aufzuchtferkeln und Mastschweinen an Rohrbreiautomaten unter dem Aspekt der Tiergerechtheit. Dissertation, Universität Hohenheim.

Kircher, A., R. Weber, B. Wechsler, und T. Jungblut (2001): Verhalten und Leistung von Aufzuchtferkeln an Rohrbreiautomaten bei unterschiedlichem Tier-Fressplatzverhältnis. In: KTBL (Hrsg.): Aktuelle Arbeiten zur artgemäßen Tierhaltung. KTBL-Schrift 403, Darmstadt, 120127. ISBN: 3-7843-2132-1.

Klindtworth, M. (1998): Untersuchungen zur automatisierten Identifizierung ovn Rindern bei der Qualitätsfleischerzeugung mit Hilfe injizierbarer 
Transponder. Forschungsbericht Agrartechnik VDI-MEG 319, Weihenstephan.

Klindtworth, M., K. Klindtworth, G. Wendl, und H. Pirkelmann (2002): Einsatz verschiedener Transpondervarianten bei Rindern (IDEA-Projekt). Landtechnik 57 (4), 230-231.

Kojima, C. J., J. A. Carroll, R. L. Matteri, K. J. Touchette, und G. L. Allee (2007): Effects of weaning and weaning weight on neuroendocrine regulators of feed intake in pigs. Journal of Animal Science 85, 2133-2139.

Kornegay, E. T., und H. R. Thomas (1981): Wet versus dry diets for weaned pigs. Journal of Animal Science 52, 14-17.

Lallés, J.-P., P. Bosi, H. Smidt, und C. R. Stokes (2007): Weaning - a challenge to gut physiologists. Livestock Production Science 108, 82-93.

Large, R. V. (1965): The artificial rearing of lambs. Journal of Agricultural Science 65: 101.

Lawlor, P. G., P. B. Lynch, G. E. Gardiner, P. J. Caffrey, und J. V. O’Doherty (2002): Effect of liquid feeding weaned pigs on growth performance to harvest. Journal of Animal Science 80, 1725-1735.

Lay, D. C., H. S. Buchanan, und M. F. Haussmann (1999): A note on simulating the "Observer Effect" using constant photoperiod on nursery pigs. Journal of Animal Science 63, 301-309.

Le Dividich, J., und B. Séve (2001): Energy requirements of the young pig. In: M. A. Varley and J. Wiseman (Hrsg.): The weaner pig - nutrition and management. CAB International, Wallingford, Oxon, UK, 17-43. ISBN: 08519-9532-2.

Lehmann, B., M. Weber (2005): Fütterungssysteme für die Ferkelaufzucht: Sensorgesteuerte Verfahren contra Breiautomaten. In: KTBL (Hrsg.): Aktuelle Arbeiten zur artgemäßen Tierhaltung. KTBL-Schrift 439, Darmstadt, 20-29. ISBN: 3-7843-2183-6.

Lexer, D., J. Baumgartner, und J. Troxler (2001): Einfluss von Gruppengröße und Gruppenzusammensetzung auf die Tagesperiodik des Verhaltens von Absetzferkeln. In: KTBL (Hrsg.): Aktuelle Arbeiten zur artgemäßen Tierhaltung. KTBL-Schrift 403, Darmstadt, 46-53. ISBN: 3-7843-2132-1.

Li, Y. Z., und L. J. Johnston (2009): Behavior and performance of pigs previously housed in large groups. Journal of Animal Science 87, 14721478.

Liu, X., J. Feng, Z. Xu, Y. Lu, und Y. Liu (2007): The effects of fermented soybean meal on growth performance and immune characteristics in weaned piglets. Turkish Journal of Veterinary and Animal Sciences 31 (5), 341-345. 
Madec, F., R. Geers, P. Vesseur, N. Kjeldsen, und T. Blaha (2001): Traceability in the pig production chain. Revue Scientifique et Technique de I'Office International 20 (2), 523-537.

Madec, F., J. Le Dividich, J. R. Pluske, und M. W. A. Verstegen (2003): Environmental requirements and housing of the weaned pig. In: J. R. Pluske, J. Le Dividich, und M. W. A. Verstegen (Hrsg.): Weaning the pig concepts and consequences. Wageningen Academic Publishers, Wageningen, The Netherlands, 377-355. ISBN: 9-0769-9817-5.

Marx, D., M. Buchholz, und R. Mertz (1988): Beziehungen zwischen Haltungstechnik und Tagesrhythmus bei früh abgesetzten Ferkeln. In: KTBL (Hrsg.): Aktuelle Arbeiten zur artgemäßen Tierhaltung. KTBLSchrift 323, Darmstadt, 9-35. ISBN: 3-7843-1766-9.

Mahan, D. C., und A. J. Lepine (1991): Effect of pig weaning weight and associated nursery feeding programs on subsequent performance to 105 kilograms body weight. Journal of Animal Science 69, 1370-1378.

Mason, S. P., S. Jarvis, und A. B. Lawrence (2003): Individual differences in responses of piglets to weaning at different ages. Applied Animal Behaviour Science 80, 117-132.

McConell, J. C., J. C. Eargle, und R. C. Waldorf (1987): Efects of weaning weight, co-mingling, group size and room temperature on pig performance. Journal of Animal Science 65, 1201-1206.

McCracken, B. A., M. E. Spurlock, M. A. Roos, F. A. Zuckermann, und H. R. Gaskins (1999): Weaning anorexia may contribute to local inflammation in the piglet small intestine. Journal of Nutrition 129, 613-619.

Mikkelsen, L. L., und B. B. Jensen (1998): Performance and microbial activity in the gastrointestinal tract of piglets fed fermented liquid feed at weaning. Journal of Animal and Feed Sciences 7, 211-215.

Miller, H. M., S. M. Carroll, F. H. Reynolds, und R. D. Slade (2007): Effect of rearing environment and age on gut devolopment of piglets at weaning. Livestock Science 108, 124-127.

Morméde, P., und M. Hay (2003): Behavioural changes and adaptions associated with weaning. In: J. R. Pluske, J. Le Dividich, and M. W. A. Verstegen (Hrsg.): Weaning the pig-concepts and consequences. Wageningen Academic Publishers, Wageningen, The Netherlands, 5357. ISBN: 9-0769-9817-5.

Newberry, R. C., und D. G. M. Wood-Gush (1986): Social relationships of piglets in a semi-natural environment. Animal Behaviour 34, 1311-1218.

Niekamp, S. R., M. A. Sutherland, G. E. Dahl, und J. L. Salak-Johnson (2007): Immune response of piglets to weaning stress: Impacts of photoperiod. Journal of Animal Science 85, 93-100. 
O'Connell, N. E., V. E. Beattie, und R. N. Weatherup (2001): Influence of group size on the performance and behaviour of 4 to 10 week old pigs. Animal Science 69, 481-489.

Orgeur, P., N. Marvic, P. Yvore, S. Bernard, R. Nowak, und F. Levy (1998): Artificial weaning in sheep: consequences on behavioural, hormonal and immuno-pathological indicators of welfare. Applied Animal Behaviour Science 58, 87-103.

Owen, F. G., und L. L. Larson (1982): A simplified liquid feeding program for calves. Journal of Dairy Science 65 (7), 1350-1356.

Pajor, E. A., D. Fraser, und D. L. Kramer (1991): Individual variation in the consumption of solid food by suckling pigs and its relationship to postweaning performance. Applied Animal Behaviour Science 32, 139-155.

Pajor, E. A., D. M. Weary, D. Fraser, und D. L. Kramer (2002): Alternative housing for sows and litters: Part 3. Effects of diet quality for piglets on pre- and postweaning behaviour and performance. Applied Animal Behaviour Science 76, 267-277.

Partridge, G. C., J. Fisher, H. Gregory, und S. G. Prior (1992): Automated wet feeding of weaner pigs vs. conventional dry diet feeding: Effect on growth rate and food consumption. Animal Production 54, 484.

Petersen, H. V., K. Vestergaard, und P. Jensen (1989): Integration of pigletes into social groups of free-ranging domestic pigs. Applied Animal Behaviour Science 23, 223-236.

Pitts, A. D., D. M. Weary, E. A. Pajor, und D. Fraser (2000): Mixing at young ages reduces fighting in unacquainted domestic pigs. Applied Animal Behaviour Science 68, 191-197.

Pluske, J. R., I. H. Williams, und F. X. Aherne (1995): Nutrition of the neonatal pig. In: M. A. Varley (Hrsg.): The neonatal pig-development and survival. CAB International, Wallingford, UK, 187-235. ISBN: 0-8519-8925-X.

Pluske, J. R., I. H. Williams, und F. X. Aherne (1996): Villous height and crypt depth in piglets in response to increases in the intake of cows'milk after weaning. Animal Science 62, 145-158.

Pluske, J. R., D. J. Hampson, und I. H. Williams (1997): Factors influencing the structure and function of the small intestine in the weaned pig: a rewiev. Livestocj Production Science 51, 215-236.

Pluske, J., J. Le Dividich, and M. W. A. Verstegen (2003): Introduction. In: J. R. Pluske, J. Le Dividich, und M. W. A. Verstegen (Hrsg.): Weaning the pig concepts and consequences. Wageningen Academic Publishers, Wageningen, The Netherlands, 15-16. ISBN: 9-0769-9817-5. 
Richtlinie 2001/91/EG (2001): Amtsblatt der Europäischen Gemeinschaften L 316, 36-38.

Schäfer, E.-M., (1999): Vergleichende Untersuchungen des Nahrungsaufnahmeverhaltens und der Wachstumsintensität von Mastschweinen und Ferkeln an Rohrbreiautomaten und anderen Fütterungssystemen unter besonderer Berücksichtigung der Gruppengröße. Dissertation, Universität Gießen.

Schlichte, R. (1999): Ferkelaufzucht in Großgruppen, Sensorfütterung, Tierverhalten und biologische Leistungen. Agrartechnische Forschung 6, 99-104.

Scholten, R., C. M. C. van der Peet-Schwering, L. A. den Hartog, J. W. Schrama, und M. W. A. Verstegen (2002): Fermented wheat in liquid diets: Effects on gastrointestinal characteristics in weanling piglets. Journal of Animal Science 80, 1179-1186.

Schrenk, H.-J. (1981): Der Einfluss von Licht und Futtergabe auf den Tagesrhythmus der Aktivität von Ferkeln. Dissertation, Universität Hohenheim.

Schrenk, H.-J., und D. Marx (1982): Der Aktivitätsrhythmus von Ferkeln und seine Beeinflussung durch Licht und Futtergabe. 2. Mitteilung: Experimentelle Untersuchungen zum Einfluss von Licht und Futtergabe. Berliner und Münchener Tierärztliche Wochenschrift 95, 61-65.

Slader, R. W., und A. M. S. Gregory (1988): An automatic feeding and weighing system for ad libitum fed pigs. Computers and Electronics in Agriculture 3, 157-170.

Spoolder, H. A. M., S. A. Edwards, und S. Corning (1999): Effects of group size and feeder space allowance on welfare in finishing pigs. Animal Science 69, 481-489.

Stangel, G., Jensen, P. (1991): Behaviour of semi-naturally kept sows and piglets (except suckling) during 10 days postpartum. Applied Animal Behaviour Science 23, 223-236.

Stolba, A., Wood-Gush, D. G. M. (1989): The behaviour of pigs in a seminatural environment. Animal Production 48, 419-425.

Tierschutz-Nutztierhaltungsverordnung (2006): Bundesgesetzblatt 2006, Teil I 41, 2043-2056.

Trevarthen, A. (2006): The national livestock identification system: The importance of traceability in E-business. Journal of Theoretical and Applied Electronic Commerce Research 2 (1), 49-62.

Tsourgiannis, C. A., P. H. Brooks, und J. Eddison (2004): Post-weaning biting, suckling and chewing behaviour of piglets weaned at 3,4 or 5 Weeks of 
age. In: Proceedings of the 38th International Congress of the International Society for Applied Ethology, Helsinki, 220.

Turnert, S. P., M. Dahlgren, D. S. Arey, und S. A. Edwards (2002): Effect of social group size and initial live eight on feeder requirements of growing pigs given food ad libitum. Animal Science 75, 75-83.

Van Beers-Schreurs, H. M. G., M. J. A. Nabuurs, L. Vallenga, H. J. Kalsbeekvan der Valk, T. Wensing, und $\mathrm{H}$. J. Breuking (1998): Weaning and the weanling diet influence the villous height and crypt depth in the small intestine of pigs and alter the concentrations of short-chain fatty acids in the large intestine and blood. Journal of Nutrition 128 (6), 947-953.

Van Putten, G. (1978): Schwein. In: H.-H. Sambraus (Hrsg.): Nutztierethologie Das Verhalten landwirtschaftlicher Nutztiere. Verlag Paul Parey, Berlin, 168-213. ISBN: 3-4896-0236-6.

Von Borell, E., D. M. Broom, Y. D. Csermel, A. A. Dijkhuizen, S. A. Edwards, P. Jensen, F. Madec, und C. Stamataris (1997): The welfare of intensively kept pigs. Report of the Scientific Veterinary Committee, European Commission.

(http://europa.eu.int/comm/dg24/health/sc/oldcomm4/previous_en.html)

Von Zerboni, H. N., und A. Grauvogel (1984): Schweine. In: H. Bogner, und A. Grauvogel (Hrsg.): Verhalten Landwirtschaftlicher Nutztiere. Verlag Eugen Ulmer, Stuttgart. ISBN: 3-8001-4345-3.

Wattanakul, W., A. H. Stewart, S. A. Edward, und P. R. English (1997): Effects of grouping piglets and changing sow location on suckling behaviour and performance. Applied Animal Behaviour Science 55, 21-35.

Weary, D. M., E. A. Pajor, M. Bonenfant, S. K. Kross, D. Fraser, und D. L. Kramer (1999): Alternative housing for sows and litters: 2. Effects of a communal piglet area on pre-and post-weaning behaviour and performance. Applied Animal Behaviour Science 65, 123-135.

Weary, D. M., J. Jasper, und M. J. Hötzel (2007): Understanding weaning distress. Applied Animal Behaviour Science 110, 24-41.

Webb, J. (2004): Tracking pork from pen to plate. Advances in Pork Production 15, 33.

Weber, R., A. Kircher, B. Wechsler, und T. Jungbluth (2002): Wie tiergerecht sind Rohrbreiautomaten in der Ferkelaufzucht? Landtechnik 2, 57-58.

Weber, R. (2004): Merkblatt zum Tier-Fressplatzverhältnis bzw. Anzahl Tiere pro Automat bei verschieden Fütterungssystemen in der Schweinehaltung. Bundesamt für Veterinärwesen (Hrsg.), Tänikon, 1-6. 
Wellock, I. J., G. C. Emmans, und I. Kyriazakis (2003): Predicting the consequences of social stressors on pig food intake and performance. Journal of Animal Science 81, 2995-3007.

Whittemore, C. T. (1998): The science and practice of pig production. $2^{\text {nd }}$ edition. Blackwell Science, Oxford, UK, 624. ISBN: 0-6320-5086-1.

Williams, I. H. (2003): Growth of the weaned pig. In: J. R. Pluske, J. Le Dividich, und M. W. A. Verstegen (Hrsg.): Weaning the pig - concepts and consequences. Wageningen Academic Publishers, Wageningen, The Netherlands, 17-35. ISBN: 9-0769-9817-5.

Wolter, B. F., und M. Ellis (2002): Impact of large group sizes on growth performance in pigs in the USA. Pig News and Information 23, 17N-20N.

Wolter, B. F., M. Ellis, S. E. Curtis, E. N. Parr, und D. M. Webel (2000): Group size and floor-space allowance can affect weanling-pig performance. Journal of Animal Science 78, 2062-2067.

Zähner, M., und E. Spiessl-Mayr (2005): Elektronische Kennzeichnung von Nutztieren. Agrarforschung 12 (2), 79-83.

Zijlstra, R. T., und T. A. Scott (2000): Feed evaluation and feed intake. Advances in Pork Production 11, 221. 


\section{$8 \quad$ Anhang}

\subsection{Lebenslauf}

\section{Zur Person}

Name:

Reiners

Vorname:

Kerstin

Geburtsdatum, -ort:

17.02.1980 in Delmenhorst

Familienstand: ledig

Staatsangehörigkeit:

deutsch

\section{Schulausbildung}

\begin{tabular}{ll}
\hline $1986-1990$ & Grundschule Delmenhorst \\
$1990-1992$ & Orientierungsstufe „Schulzentrum West“, \\
& Delmenhorst \\
& $\begin{array}{l}\text { Gymnasium an der Willmsstraße, Delmenhorst; } \\
1992-1999\end{array}$ \\
& Abschluss: Abitur
\end{tabular}

\section{Landwirtschaftliche Ausbildung}

$1999-2000$

landwirtschaftlicher Betrieb Alfons Braig, 89584 Altbierlingen; Abschluss: Praktikantenprüfung (Note: 1,8)

\section{Studium}

Okt. 2000-Sept. 2002

Okt. 2002 - März 2004

Mai 2004

April 2004 - Febr. 2005

Aug. 2005
Grundstudium der Agrarwissenschaften an der Georg-August-Universität Göttingen

Studium der Agrarwissenschaften an der GeorgAugust-Universität Göttingen, Studienrichtung: Agribusiness

Bachelor-Abschluss in der Studienrichtung Agribusiness (Note: 1,8)

Studium der Agrarwissenschaften an der GeorgAugust-Universität, Studienrichtung: Tierproduktion

Master-Abschluss in der Studienrichtung Tierproduktion (Note: 1,6) 


\section{Studienbegleitende Tätigkeiten}

April 2001 - Okt. 2004

Mitarbeit in der Fachschaft der Fakultät für

Agrarwissenschaften an der Georg-August-

Universität Göttingen

Juni 2004 - Juli 2004

Beschäftigung als studentische Hilfskraft am Institut für Pflanzenpathologie und

Pflanzenschutz der Georg-August-Universität

Göttingen, Entomologische Abteilung

\section{Praktika und weitere Tätigkeiten}

Febr. 2001 - April 2001

landwirtschaftlicher Betrieb Hakemann GbR, Hasbergen

Aug. 2001 - Okt. 2001

landwirtschaftlicher Betrieb John und Susan Garner, Ontario, Kanada

Febr. 2002 - März 2002

Landwirtschaftskammer Weser-Ems, Fachbereich Tierzucht und Tierhaltung

Aug. 2002 - Okt. 2002 landwirtschaftlicher Betrieb Gut Niederelsungen, Wolfhagen

März 2003 - April 2003

Niedersächsische Erzeugergemeinschaft für Zuchtschweine, Hauptgeschäftsstelle Oldenburg

Aug. 2003 - Okt. 2003 landwirtschaftlicher Betrieb Niels Jørgen Pedersen, Ølstrup, Dänemark

Juli 2004 - Aug. 2004 landwirtschaftlicher Betrieb Niels Jørgen Pedersen, Ølstrup, Dänemark

Sept. 2004

Luxcontrol GmbH, Hamburg

\section{Beruflicher Werdegang}

Sept. 2005 - Dez. 2007

Wissenschaftliche Mitarbeiterin/Doktorandin am Forschungs- und Studienzentrum für Veredelungswirtschaft Weser-Ems der GeorgAugust-Universität Göttingen in Vechta; finanziert durch ein Stipendium der H. Wilhelm Schaumann Stiftung

Seit Sept. 2007

PIC Deutschland GmbH in Schleswig, Technical Service 


\subsection{Veröffentlichungen}

\subsubsection{Wissenschaftliche Veröffentlichungen mit Begutachtung}

Hessel, E. F., K. Reiners, und H. F. A. Van den Weghe (2006): Socializing piglets before weaning: Effects on behavior of lactating sows, pre- and postweaning behavior, and performance of piglets. Journal of Animal Science 84, 2847-2855.

Werner, C., K. Reiners, und M. Wicke (2006): Short as well as long transport duration can affect the welfare of slaughter pigs. Animal Welfare 16, 385389.

Hessel, E. F., K. Reiners, und H. F. A. Van den Weghe (2007): Verbesserung der Aufzuchtleistung von Ferkeln durch eine Modifizierung des Haltungssystems während der Säugephase. Agrartechnische Forschung 13 (4), $110-118$.

Reiners, K., E. F. Hessel, und H. F. A. Van den Weghe (2008): The effect of heated mash on performance and feeding behavior of newly weaned piglets. Journal of Animal Science 86, 3600-3607.

Reiners, K., A. Hegger, E. F. Hessel, S. Böck, G. Wendl, und H. F. A. Van den Weghe (2009): Application of RFID technology using passive HF transponders for the individual identification of weaned piglets at the feed trough. Computers and Electronics in Agriculture 68, 178-184.

Reiners, K., E. F. Hessel, S. Sieling, und H. F. A. Van den Weghe (2009): Influence of photoperiod on behavior and performance of newly weaned piglets with a focus on feeding behavior, feed consumption and growth. Journal of Swine Health and Production, eingereicht.

\subsubsection{Tagungsbände}

Reiners, K., E. F. Hessel, und H. F. A. Van den Weghe (2005): Evaluierung prozesstechnischer Einflussfaktoren auf das Futteraufnahmeverhalten und die Leistung von Absatzferkeln. Vortragstagung der DGfZ und GfT, 21.-22.09.2005, Berlin. Polykopie.

Reiners, K., E. F. Hessel, und H. F. A. Van den Weghe (2007): Einfluss des Futterbreis auf die Wachstumsleistung und das Futteraufnahmeverhalten von früh abgesetzten Ferkeln. Vortragstagung der DGfZ und GfT, 26.-27. 09.2007, Hohenheim. Polykopie.

Reiners, K., E. F. Hessel, A. Hegger, S. Böck, S. Thurner, G. Wendl, und H. F. A. Van den Weghe (2007): Evaluierung prozesstechnischer Einflussfaktoren auf die Futteraufnahme von früh abgesetzten Ferkeln mit Hilfe einer simultanen Einzeltiererkennung am Fressplatz. In: KTBL (Hrsg.): 8. Tagung Bau, Technik und Umwelt in der landwirtschaftlichen 
Nutztierhaltung, 8.-10. 10.2007, Bonn. KTBL-Schriftenvertrieb, Darmstadt, 252-257.

Hessel, E. F., K. Reiners, und H. F. A. Van den Weghe (2007): Sozialisierung von Saugferkeln: Tierverhalten und Tierleistung vor und nach dem Absetzen. In: KTBL (Hrsg.): 8. Tagung Bau, Technik und Umwelt in der landwirtschaftlichen Nutztierhaltung. KTBL-Schriftenvertrieb, Darmstadt, 114-119.

Thurner, S., G. Wendl, S. Böck, E. F. Hessel, K. Reiners, und H. F. A. Van den Weghe (2007): Reading performance of high frequency transponders under laboratory conditions and in feed suppliers for weaned piglets. In: Poster Papers Proceedings of the $6^{\text {th }}$ European Conference on Precision Agriculture and the $3^{\text {rd }}$ European Conference on Precision Livestock Farming (CD), Skiathos, Greece.

Kemper N., I. Gerjets, S. Kruse, J. Krieter, I. Diehl, C. Ewers, L. H. Wieler, und K. Reiners (2009): The role of Escherichia coli and other pathogens in mastitis in sows. XIV. International Congress in Animal Hygiene (ISAH), 19.-23. Juli 2009, Vechta. Accepted, proceedings in press.

Gerjets I., K. Reiners, und N. Kemper (2009): Study on the pathogen spectrum and analysis of risk factors for MMA in sows. XIV. International Congress in Animal Hygiene (ISAH), 19.-23. Juli 2009, Vechta. Accepted, proceedings in press.

Gerjets I., K. Reiners, und N. Kemper (2009): Analysis of the pathogen spectrum and risk factors associated with coliform mastitis in sows. 60th EAAP, 24.-27. August 2009, Barcelona, Spanien. Accepted, proceedings in press.

\subsubsection{Sonstige Veröffentlichungen}

Werner, C., K. Reiners, und M. Wicke (2005): Transportbedingte Einflussfaktoren auf die Sterblichkeit von Schlachtschweinen. Fleischwirtschaft 9, 133-136.

Reiners, K., E. F. Hessel, und H. F. A. Van den Weghe (2007): Öffnen von Buchtentrennwänden bei der Haltung von ferkelführenden Sauen im Kastenstand - Tierleistung und Tierverhalten im Vergleich zum konventionellen System. Landtechnik 62 (2), 104-105.

Hessel, E. F., K. Reiners, A. Hegger, S. Böck, G. Wendl, und H. F. A. Van den Weghe (2008): Simultane Einzeltiererkennung - Einsatz der RFIDTechnologie unter Verwendung von HF-Transpondern. Landtechnik 63 (1), 38-39. 


\section{Danksagung}

Mein herzlicher Dank gilt allen, die auf ihre Art und Weise zum Gelingen dieser Arbeit beigetragen haben.

Herrn Prof. Dr. Ir. H. Van den Weghe danke ich für die Überlassung des Themas, die hilfreichen Verbesserungsvorschläge und die Unterstützung bei der Anfertigung der Arbeit.

Bei Frau PD Dr. Engel Hessel bedanke ich mich für die Diskussionsbereitschaft und die Unterstützung bei der statistischen Auswertung der Daten und der Veröffentlichung der Ergebnisse.

Herrn Prof. Dr. Michael Wicke danke ich herzlich für die Übernahme des Korreferats und seine motivierenden Worte in der Endphase.

Der $\mathrm{H}$. Wilhelm Schaumann Stiftung sei an dieser Stelle für die finanzielle Unterstützung gedankt.

Ein herzliches Dankeschön geht an Heinrich Dierckes und Bernadette von Lehmden für die unkomplizierte Lieferung der Ferkel.

Herrn Peter Enders möchte ich für die zur Verfügung gestellten PreMixer und die gewährte Diskussionsbereitschaft in allen Bereichen der Fütterungstechnik danken.

Bei der Firma Big Dutchman, namentlich bei Herrn Dr. Gnosa, bedanke ich mich für die Bereitstellung der Lean Machines.

Ein herzlicher Dank gebührt Herrn Dr. Georg Wendl, Stefan Böck und Stefan Thurner vom Institut für Landtechnik und Tierhaltung der Bayerischen Landesanstalt für Landwirtschaft, für die Entwicklung der simultanen Einzeltiererkennung und die jederzeit entgegengebrachte Unterstützung bei allen Fragen rund um die Technik.

Ein besonders großes Dankeschön geht an die Familie Fleming, die die Tiere nach Versuchsende am Forschungszentrum gemästet hat und uns trotz unserer morgendlichen Überfälle mit der Waage jederzeit fröhlich empfangen und unterstützt hat.

Den Mitarbeitern des Forschungs- und Studienzentrums für Veredelungswirtschaft in Vechta danke ich für die schöne Zeit am Institut und für die Unterstützung bei der Durchführung der Versuche. Besonderer Dank gilt hierbei Heinz Siebenand für sein großes Einfallsreichtum in allen stallbaulichen Belangen, Hartmut Liebenow für seine "goldenen Hände“, mit denen er auch die hoffnungslosesten Fälle der Mess- und Videotechnik zum Leben erwecken konnte, Uwe Vehlow für die Bewältigung der regelrechten Datenflut bei der Einzeltiererkennung, Hans-Jürgen Technow für die zuverlässige Unterstützung bei der Versorgung der Tiere und Dr. Manfred Kaiser und Barbara Thomann für ein paar ferkelfreie Wochenenden.

Meinen ehemaligen „Buchtengenossen“ Manuela Olberding, Jörgen Haneke, Felix Garlipp, und ganz besonders Kathrin Fleming danke ich für eine unvergessliche und lustige Zeit, die vielen aufmunternden Gespräche und dafür, dass sie mich auch mit „schweinischer“ Duftnote zu jeder Zeit in ihrer Mitte ertragen haben. 
Franziska Kubitza, Mareike Fleming, Alexander Hegger und Stefan Sieling möchte ich für die zahlreichen Stunden vor dem Fernseher bei der Auswertung der Verhaltensbeobachtungen danken.

Barbara Berger danke ich für einen Samstagnachmittag voller „Formatierungswunder“.

Birthe Lassen danke ich für Ihre unermüdlichen Motivationsversuche, die „Survival-Pakete“, und die Last-Minute-Korrekturdienste.

Ein ganz lieber Dank geht an Yogi, Caro, Tine, Viola, Steffi und Nina für ihre geduldigen und immer offenen Ohren und die vielen Aufmunterungsversuche auch in "hoffnungslosen“ Situationen... Ihr seid die besten Freunde der Welt!

Der allergrößte Dank gebührt jedoch meinen Eltern und Markus.

Markus danke ich für sein Verständnis, seinen Rückhalt, und seine starken Nerven, mit denen er so manche Doktorarbeitslaune geduldig ertragen hat.

Meinen Eltern danke ich von ganzem Herzen für ihre große Unterstützung während meiner gesamten Ausbildung und dafür, dass sie zu jeder Zeit an mich glauben und immer für mich da sind. Ihnen ist diese Arbeit gewidmet. 